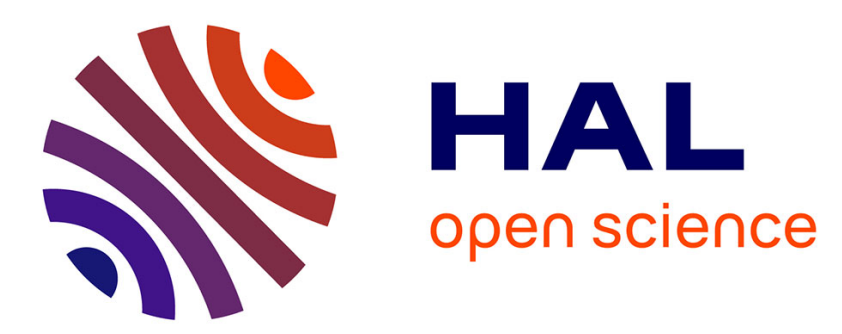

\title{
A three dimensional numerical quantum mechanical model of electronic field emission from metallic surfaces with nanoscale corrugation
}

Bruno Lepetit

\section{- To cite this version:}

Bruno Lepetit. A three dimensional numerical quantum mechanical model of electronic field emission from metallic surfaces with nanoscale corrugation. Journal of Applied Physics, 2019, 125 (2), pp.025107. 10.1063/1.5063901 . hal-01982362

\section{HAL Id: hal-01982362 \\ https://hal.science/hal-01982362}

Submitted on 15 Jan 2019

HAL is a multi-disciplinary open access archive for the deposit and dissemination of scientific research documents, whether they are published or not. The documents may come from teaching and research institutions in France or abroad, or from public or private research centers.
L'archive ouverte pluridisciplinaire HAL, est destinée au dépôt et à la diffusion de documents scientifiques de niveau recherche, publiés ou non, émanant des établissements d'enseignement et de recherche français ou étrangers, des laboratoires publics ou privés. 
This manuscript was accepted by J. Appl. Phys. Click here to see the version of record.

\title{
Publishing A three dimensional numerical quantum mechanical model of electronic field emission from metallic surfaces with nanoscale corrugation.
}

\author{
Bruno Lepetit* \\ Laboratoire Collisions Agrégats Réactivité, \\ UMR5589, Université de Toulouse, CNRS, \\ Bât. 3R1b4 - 118 route de Narbonne 31062 Toulouse Cedex 09, France
}

(Dated: November 27,2018)

\begin{abstract}
The effects on the electronic emission of the presence of nanoscale steps on a tungsten surface are investigated for the first time using three dimensional quantum mechanical models. The plane wave periodic version of the density functional theory is used to obtain the electronic wavefunctions and potentials for flat and corrugated structures. Local and averaged emitted current densities are obtained from them using time dependent perturbation theory. The orders of magnitude of the averaged current densities resulting from these calculations are similar for both flat and corrugated cases, however, strong enhancements are observed on the local current densities near the edges of the steps. These numerical results are compared with those of the analytical Fowler-Nordheim type models. The slopes of the Fowler-Nordheim plots are in good agreement for both numerical and analytical models, but the magnitudes of the emitted currents are significantly different. This is related to weaknesses in the description of the electronic structure of the metal in the analytical models.
\end{abstract}




\section{Publishing INTRODUCTION}

There has been recently a revival of interest in the nuclear fusion industry community ${ }^{1,2}$ on the problem of electronic emission induced by static electric field ${ }^{3,4}$ because it can cause electrical breakdown in vacuum. This poses challenges to the design of vacuum insulation structures in high voltage systems. These are parts of the heating systems of magnetically confined plasmas in tokamaks which are based on high energy hydrogen or deuterium atoms accelerated by the electric field of high voltage systems. Unfortunately, the performances of such heating systems are limited by damaging electron currents induced by field emission ${ }^{1,5-7}$. These currents can be reduced by raising the pressure in the vacuum system, typically from ultrahigh vacuum to pressures of the order of $10^{-4}=10^{-2} \mathrm{~Pa}^{8-14}$. This gas effect, known for quite some time, has been investigated recently in details for tungsten carbide and tungsten cathodes ${ }^{15-20}$. Accurate first principles emission models are required to understand better the correlations between emission properties and changes induced on the surface by the presence of the ambient gas. Unfortunately, most of the models currently available are too crude to provide such detailed information

Indeed, our understanding of electronic emission still relies mainly on the historical Fowler-Nordheim model ${ }^{3,21}$, corrected in ref. 22 , extented to thermo-ionic emission by Murphy and Good in ref. 4, to curved emitters in ref. 23. A generalization using the local density of states at the surface has been proposed in ref. 24. Convenient simplifications of the analytical model have been given in ref. 25 and a universal formulation in ref. 26. We refer to these models globally as FN type models, sometimes distinguishing within them between the FN model (the historical Fowler-Nordheim one ${ }^{3,21}$ ) and the MG one (Murphy and $\operatorname{Good}^{4}$ ). It has been used widely and successfully over the years as a fitting model of experimental data, recent examples of applications are given in the citations of ref. 27. However, this model relies on a crude description of the emitting electrode by a single parameter, the work function. It is more a semi-empirical model than an ab initio one and it cannot correlate emission to surface structure details such as the presence of defects or adsorbates.

More quantitative models have been designed recently, in particular in the context of the development of carbon based nanomaterials and two-dimensional materials (for reviews, see ref. 28,29). Some are improvements of the FN analytical models ${ }^{30,31}$. Others are based 
Publishimg Density Functional Theory (DFT) calculation of the Hartree and exchange-correlation potentials which drive electron emission, followed by emitted electron time dependent ${ }^{20,32-34}$ or time independent ${ }^{35-45}$ dynamical models. In particular, we presented in a recent paper ${ }^{20}$ a method using plane wave DFT followed by electronic wavepacket time propagation within the perturbation theory framework to compute currents emitted from structures modeled atom-by-atom. This method was tested on the emission from flat-tungsten.

It is well known ${ }^{8,46}$ that surface corrugation enhances locally the external electric field and thus emission levels. Recently, FN type models have been extended to such systems ${ }^{47}$. Typically, local field enhancement factor $\beta$ of several hundreds have been obtained for unpolished stainless steel ${ }^{48}$ or copper ${ }^{49}$ surfaces, whereas $\beta$ of several tens have been measured for titanium, molybdenium ${ }^{50}$ and niobium ${ }^{51}$, for rugosities-of the order of ten nanometers or more. The role of protrusions has already been considered from a theoretical point of view (for a review see for instance ref. 52). In a recent study ${ }^{19}$, we considered from a theoretical point of view the correlations between surface corrugation at the nanoscale and field enhancement. In the present paper, we want to go further by addressing the more difficult correlations between corrugation and emission enhancement. To our knowledge, the present study represents the first application of DFT-type models beyond flat structures on corrugation in the field emission context. We describe in section II the computational method which we have implemented. We present in sub-section II A the formalism and we explain how to use the potentials and the states resulting from a DFT calculation to propagate a wavepacket in time within perturbation theory. Then, in sub-section II B, we provide details about the computational implementation of this formalism. In section III, we show the results of the implementation of the method for a W surface, either flat or corrugated by nano-scale steps. The comparison between both calculations allows to obtain information on the effect of corrugation on emission. We also compare the results of our model with the ones resulting from the FN type methods. 


\section{COMPUTATIONAL METHOD}

\section{A. General framework}

We consider the electrons of a metallic slab at $0 \mathrm{~K}$ temperature. This slab is infinite in the $x$ and $y$ directions but it is not necessarily of constant thickness in $z$ and can present periodic features. Such features can be for instance periodic steps on the surface or adsorbates. This slab is subjected to an external field $\mathbf{F}$ parallel to $z$ which induces an electronic current flowing from the metal into the vacuum. Bold-face symbols refer to vectors, the corresponding standard symbols refer to their norms. We assume that this current is weak enough so that the perturbation theory can be used to model accurately this process and we use its time dependent version.

We assume that the electrons occupy orbitals $\Psi_{m}(\mathbf{r}, \mathbf{F}=0)$ which are the Bloch functions (p. 179 in ref. 53) defined by :

$$
(T+U(\mathbf{r}, \mathbf{F}=\mathbf{0})) \Psi_{m}(\mathbf{r}, \mathbf{F}=0)=\epsilon_{m} \Psi_{m}(\mathbf{r}, \mathbf{F}=0)
$$

$T$ is the electronic kinetic energy operator and $U(\mathbf{r}, \mathbf{F}=\mathbf{0})$ is the total potential energy in the absence of external field experienced by an electron of the material located at $\mathbf{r}$. $m$ is a composite index which comprises the electron momentum in the first Brillouin zone (p. 37 in ref. 53) and the band number. Periodic conditions are applied at the boundary of a volume $\Omega$ consisting of $N_{k}$ unit cells, where $N_{k}$ is the number of k-points used to sample the first Brillouin zone. The orbitals are normalized such that they correspond to one electron charge over $\Omega$.

When the external field is applied, the electrons experience a potential $U(\mathbf{r}, \mathbf{F})$ given by : $U(\mathbf{r}, \mathbf{F})=U(\mathbf{r}, \mathbf{F}=\mathbf{0})+\Delta U(\mathbf{r}, \mathbf{F}) . \Delta U(\mathbf{r}, \mathbf{F})$ is the perturbation associated with the external field : it vanishes inside the metal and it is linear far away from the metal. In practice, we observe stability problems in the DFT calculation at large field. Therefore, we compute the potential for a lower reference field $\mathbf{F}_{\mathbf{0}}$ (typically $1 \mathrm{~V} / \mathrm{nm}$ ) and we obtain $\Delta U(\mathbf{r}, \mathbf{F})$ by linear extrapolation : $\Delta U(\mathbf{r}, \mathbf{F})=\frac{F}{F_{0}} \Delta U\left(\mathbf{r}, \mathbf{F}_{\mathbf{0}}\right)$.

As a result of this perturbation, metal electrons can tunnel and leak into the vacuum. The leakage rate of each orbital is a "state current" $I_{m}$ given within the frame of the time 
Publishidgendent perturbation theory as the Fourier transform of the correlation function $C_{m}(t)$ :

$$
\begin{gathered}
I_{m}=\frac{e}{\hbar^{2}} \int_{-\infty}^{+\infty} d t e^{i \frac{\epsilon_{m} t}{\hbar}} C_{m}(t) \\
C_{m}(t)=<\Phi_{m}\left|e^{-i \frac{T+\Delta U(\mathbf{r}, \mathbf{F})+U_{v a c}}{\hbar}}\right| \Phi_{m}>
\end{gathered}
$$

$U_{v a c}$ is the vacuum potential (the work function if the 0-energy is the Fermi energy), $e$ the electron charge, $\hbar$ the reduced Planck constant. The correlation function $C_{m}(t)$ is a matrix element of the evolution operator associated to the perturbing potential. It involves the state $\mid \Phi_{m}(\mathbf{r}, \mathbf{F})>$ which results from the action of the perturbation on the field-free electronic orbitals : $\left|\Phi_{m}(\mathbf{r}, \mathbf{F})>=\Delta U(\mathbf{r}, \mathbf{F})\right| \Psi_{m}(\mathbf{r}, \mathbf{F}=0)>\cdot\left(\mid \Phi_{m}(\mathbf{r}, \mathbf{F})>\right.$ is expelled quickly into vacuum and the correlation function $C_{m}(t)$ decreases quickly to 0 , allowing for a converged Fourier transform (eq. 2) on a short time window (typically of the order of a few 10 fs). However, on some occasions, a tail of the wevapacket remains stuck in the metal region, enlarging Fourier time window and slowing down computing speed. We therefore added a repulsive potential in the metallic region to restore fast convergence, and we checked that this modification does not significantly change the current values.

The total current emitted by $\Omega$ is : $I=2 \sum_{m} I_{m}$ (the factor 2 is due to spin degeneracy) and the corresponding averaged current density is : $\bar{J}=\frac{I}{N_{k} A_{u c}}$, where $A_{u c}$ is the emitting area of the unit cell. An averaged current density $\overline{J_{m}}$ can be defined similarly from $I_{m}$ for each state $\Psi_{m}(\mathbf{r}, \mathbf{F}=0)$.

We can also define local current densities $J(x, y)$ from the correlation function $C_{m}(t)$. Indeed, is obtained the 3 dimensional spatial quadrature which defines $C_{m}(t)$ in eq. 3 can separated in a 1 dimensional quadrature in $z$ and in 2 dimensional one in $x, y$ quadratures. To do so, we introduce a local correlation function $c_{m}(x, y, t)$ :

$$
\begin{gathered}
c_{m}(x, y, t)=\int d z \Phi_{m}^{*}(\mathbf{r}, \mathbf{F}) e^{-i \frac{T+\Delta U(\mathbf{r}, \mathbf{F})+U_{v a c}}{\hbar} t} \Phi_{m}(\mathbf{r}) \\
C_{m}(t)=\int d x \int d y c_{m}(x, y, t)
\end{gathered}
$$

A local state current density $J_{m}(x, y)$ can be obtained from the Fourier transform of $c_{m}(x, y, t):$

$$
J_{m}(x, y)=\frac{e}{\hbar^{2}} \int_{-\infty}^{+\infty} d t e^{i \frac{\epsilon m t}{\hbar}} c_{m}(x, y, t)
$$


Publishinge total local current density is then : $J(x, y)=2 \sum_{m} J_{m}(x, y)$. It is easy to check that : $\bar{J}=\frac{\int d x \int d y J(x, y)}{N_{k} A_{u c}}$, as expected.

The present formalism has similarities with the Bardeen Transfer Hamiltonian (BTH) model of tunneling ${ }^{54}$ because it also relies on a perturbative formalism. The BTH formalism is an ingredient of the Tersoff-Haman mode ${ }^{55}$ used in the context of scanning tunneling microscopy to obtain current density maps. Similarly, local density $\mathcal{J}(x, y)$ maps can be obtained from the present formalism in the context of electronic field emission. Current maps obtained by application of this new formalism will be-presented below.

\section{B. Numerical implementation}

We now illustrate the use of our method on bcc tungsten, and we consider emission from flat (001) surfaces as well as corrugated ones. Tungsten is a commonly used material for electrodes and has already been the subject of numerous studies using DFT (see our recent work, ref. 17-20 and references therein). Qur previous results concerning electronic emission include the effect of carbon adsorption ${ }^{17}$ in the context of experiments ${ }^{18}$. The effect of the nano-corrugation of the surface on Jocal field enhancement was considered in ref. 19 and its effect on field emission is the focus of the present paper.

The flat structure considered here is shown on fig. 1. It is a slab of 7 atomic planes bounded on both sides by a (001) surface. Its thickness is $3 a$, where $a$ is the lattice parameter. The slab is submitted on both side to the same static electric field parallel to $z$, so that one of the 2 surfaces is the cathode and simultaneously the other one is the anode. Using such symetric structures is important for the DFT calculation to converge rapidly with respect to the supercell size along z (see section 4.7 in ref. 56). Following our previous work $^{17}$, the lattice parameter is chosen as $a=3.179 \AA$ and the work function is $\varphi=4.25 \mathrm{eV}$. The corrugated structure considered here is shown on fig. 2. It is obtained from bulk bcc W cut at $45^{\circ}$ along the (101) plane. The surface consists of $3(001) \times 3(100)$ steps-terraces, this is a simple generic form of corrugation at the nanoscale. The corrugation is periodic, with periods $b=3 \sqrt{2} a$ and $a$ along $x$ and $y$, respectively. It defines ridge and trough lines parallel to the $y$ axis. As for the flat case, the structure is symetrized to ease numerical convergence, one of its side is a corrugated cathode and the other one is the anode. The electric field is applied along an axis perpendicular to the (101) plane, chosen as the $z$ axis. 
Publishinge structure shown on fig. 2 is the symetrized structure with minimum thickness to reduce the length of the computations. From our DFT calculations (see below), its work function is $4.02 \mathrm{eV}$. On fig. 1 and 2 , the $x, y$ origin is left front corner.

The ab initio total-energy and molecular dynamics program VASP (Vienna Ab initio Simulation Program) developed at the Institut für Materialphysik of the Universität Wien has been used for all DFT calculations ${ }^{57-60}$. We use this program in a way very similar to the one already described in refs. 17,19,20. The electron-ion interaction for tungsten is described by the projector augmented wave potential $(\mathrm{PAW})^{61,62}$. The exchange-correlation energy is calculated within the generalized gradient approximation (GGA) using the revised form of the Perdew, Burke, and Ernzerhof functional (PBE) ${ }^{63-65}$ We consider six $5 d 6 s$ valence electrons for each tungsten atom. Fractional occupancies are-calculated using a second-order Methfessel-Paxton smearing function ${ }^{66}$ with a width of $0.2 \mathrm{eV}$. All plane waves of the basis set are expanded up to a kinetic energy cutoff of $350 \mathrm{eV}$. For the flat case, the use a supercell of size $(a, a, 26 a)$ along $(x, y, z)$ and a $(16 \times 16 \times 1)$ k-point grid. For the corrugated case, the supercell size is $(3 \sqrt{2} a, a, 14 a)$ and the k-point grid is reduced to $(6 \times 16 \times 1)$ as the structure period along $x$ is larger than along $y$. In presence of the external field, an artificial dipole parallel to $z$ must be added in the vacuum region to allow for periodicity of the electrostatic potential between the borders of the supercell.

The VASP calculation provides two kinds of physical quantities necessary for the emitted current calculation. The first one is the Kohn-Sham orbitals $\Psi_{m}(\mathbf{r}, \mathbf{F}=0)$, see eq. 1. They are provided in VASR by their expansion on a plane-wave basis. We obtained the values of these orbitals on a 3D spatial grid using our implementation based on fast Fourier transforms of the post-processing Wavetrans ${ }^{67}$. Moreover, we use in the following the projections of these orbitals on local spd atomic orbitals which are atom centered spherical harmonics to analyze physically the results of our computations.

The second kind of physical quantities provided by VASP are the potentials $\Delta U(\mathbf{r}, \mathbf{F})$, for zero and non-zero fields. The electric fields are obtained straightforwardly these potentials by derivation in the Fourier space. The potentials obtained from the present DFT approach suffer from a serious shortcoming, which is that charge-image interaction is not taken into account properly : in the flat case, in the absence of external field, the potential has an exponentially decreasing character instead of a Coulombic $-\frac{1}{16 \pi \varepsilon_{0} z}$ one near the metal in vacuum. This deficiency is common to all DFT approaches which are local or semi-local. 
Publishinign ple phenomenological fixes can be implemented in the flat case : they consist in switching smoothly from the DFT potential inside the metal to the standard analytical charge-image interaction in vacuum ${ }^{68-70}$. It is not clear however how to implement such fixes in the corrugated case. More rigorously, one could use fully non local DFT approaches, like the

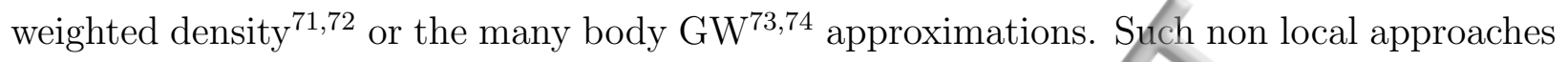
however lead to extremely difficult and lengthy calculations, beyond currently available computational ressources for the large systems considered here. We therefore use in the present study the semi-local PBE approach. Although the-absolute values of the currents may require corrections, we believe that the comparison of the relative emission properties of flat and corrugated surfaces presented here is meaningful.

Moreover, we have observed sometimes convergence problems in VASP at large fields, as already described in ref. 20. The same linear extrapolation method from low field calculations which converge easily to large field ones has been used.

The correlation functions defined in eq. 3 are obtained by time propagation of the modified Kohn-Sham orbitals $\mid \Phi_{m}(\mathbf{r}, \mathbf{F})>$ using the $2^{\text {nd }}$ order split operator method ${ }^{75}$. Typically, the states are propagated in time up to $20 \mathrm{fs}$ with 400 steps. The total current is obtained by summation over the states which have a non negligible decay rate, they are expected to have energies within a limited band around the Fermi energy. We could achieve convergence by using the interval $[-1.5 \mathrm{eV}, 0.2 \mathrm{eV}]$ (the 0 energy being the Fermi energy), for both the flat and corrugated cases. For the flat case, there are 1372 states inside this band, taking into account the degeneracies induced by the symmetries of the k-point space allows to propagate only 192 states out of them. For the corrugated case, there are 2180 states in this band, in this case the k-point space has a lower symmetry and 545 states have to be propagated.

\section{RESULTS}

Fig. 3 shows the $z$ component of the induced electric field along $z$, for selected $x, y$ locations on the flat and corrugated surfaces. These fields have characteristics consistent with those already observed in ref. 19. In the flat case, the field has, as expected, little dependence on the surface $x, y$ location. It decreases from its asymptotic value to 0 on a layer 2-3 $\AA$ thick above the topmost atoms, due to the screening of the external field by the delocalized metal electrons. For the corrugated case, also as expected, the field is enhanced 
Publishingthe ridge region and expelled from the trough one. The enhancement factor is maximum near $3 \AA$ above the ridge and reaches $\beta \approx 1.5$. This result is quantitatively consistent with the result of ref. 19 which provides a simple power law for the enhancement near right angle ridges : $\beta \approx\left(\frac{9}{20 \bar{z}}\right)^{\frac{1}{3}}$ as a function of a reduced distance $\bar{z}=\frac{2\left(z-z_{i m}\right)}{b}$. $z_{i m}$ corresponds to the repulsion distance and is the barycenter of the induced charge, typically ${ }^{19}: z_{i m}=2.13 \AA$, so that $\bar{z} \approx 0.13$ at the maximum enhancement. This model provides: $\beta \approx 1.51$, in excellent agreement with the present numerical result.

Fig. 4 and fig. 5 are images of the local current density emitted by the unit surface cell for an external field at large distance $F=15 \mathrm{~V} / \mathrm{nm}$. The current density is scaled by $F^{2}$ for consistency with the Fowler-Nordheim plot which will be presented on fig. 6. In most cases, images are presented in the context of Scanning Tunneling Microscopy (STM) and are obtained with the help of the Tersoff-Haman model ${ }^{55}$. Assuming a localized tip wave function, the tunneling current becomes directly proportional to the local density of states (LDOS) at the Fermi level of the sample under study. A 2D image is obtained by plotting the LDOS on a plane located at an appropriate height above the surface LDOS, typically the height of the tip above the sample. Our present model is specific to field emission and is free from such an adjustable parameter, indeed the anode which collects electrons is set at infinity.

In the flat case (fig. 4), the topmost layer (layer \# 7) atom is at the center of the figure $(x, y=a / 2, a / 2)$, atoms belonging to layer \# 6 are at the corners. The highest emission point is located at the topmost atom, lowest emission points are at the layer \# 6 atom locations. However, the contrast between low and high emission is limited, less than $7 \%$, as expected for a flat surface. The averaged current density is $1.69 \times 10^{-5} \mathrm{~A} / \mathrm{nm}^{2}$. Fig. 5 corresponds to the corrugated case. The ridge line is located at $x=\frac{b}{2}$ and the troughs at $x=0$ and $x=b$. The atoms facing vacuum are depicted by disks increasingly larger and darker as their height increases. There is now a strong dependence of the emitted current density on the $x, y$ location, the density at the ridge is more than one order of magnitude larger than at the troughs. Interestingly, although atoms from both layers $\# 7$ (at $x, y=b / 2,0$ and $x, y=b / 2, a)$ and $\# 6$ (at $x, y=b / 2, a / 2$ ) are located on the ridge, only limited variations of the current density are observed along this line. In fact, the current density is found slightly larger in the vicinity of the atom at the center of the cell which belongs to layer \#6 than in the vicinity of the atoms belonging to layer \# 7 (at $x, y=b / 2,0$ and $x, y=b / 2, a$ ). The 
Publishing aged emitted current density $\bar{J}$ is $1.81 \times 10^{-5} \mathrm{~A} / \mathrm{nm}^{2}$, which is only $7 \%$ larger than the emitted current density in the flat case. Although corrugation induces field and electronic emission enhancements locally, it barely modifies global emission from the surface.

Fig. 6 is a Fowler-Nordheim plot which compiles our numerical results for different field strengths. The averaged current density $\bar{J}$ is shown for the flat and corrugated cases (full lines without markers). In the corrugated case, the current density depends strongly on the emission points. It is shown for the trough $(x, y=0,0)$ and ridge $(x, y=b / 2, a / 2)$ locations as dashed lines connecting, respectively, upward and downward pointing triangles. The scaled current densities represented in lin-log scale as a function of the inverse field have the usual linear behavior typical of the Fowler-Nordheim model, with the addition of a saturation effect visible above $15 \mathrm{~V} / \mathrm{nm}$ in the corrugated ease. Interestingly, this saturation does not appear in the flat case nor in the vicinity of the trough for the corrugated case. In the latter case, it appears on the ridge current density which it is very high in this area as well as on the averaged density, as a consequence of the important contribution of the ridge to it. A similar saturation effect at large field has also been observed in other emission models. One of them is the simple self consistent one dimensional DFT calculation performed with a local exchange-correlation functional where the metal is modeled by a jellium, i.e. where the nuclei localized positive charges are replaced by a uniform distribution (fig. 4 in ref. 37).

The present results can be compared with those of the Fowler-Nordheim $(\mathrm{FN})^{3,21,22}$ and Murphy-Good $(\mathrm{MG})^{4} /$ models. In both cases, the current density is independent of the $x, y$ location and is obtained as

$$
J=\frac{e^{3}(\beta F)^{2}}{16 \pi^{2} \hbar \varphi\left(t\left(y_{F}\right)\right)^{2}} \exp \left(-\frac{4}{3 e}\left(\frac{2 m}{\hbar^{2}}\right)^{\frac{1}{2}} \frac{\varphi^{\frac{3}{2}} v\left(y_{F}\right)}{\beta F}\right)
$$

In the FN model $v(y)=1$; in the MG model, it is a function of the variable $y=\frac{\left(\frac{e^{3} \beta F}{4 \pi \sigma_{0}}\right)^{\frac{1}{2}}}{U_{v a c}-\epsilon}$ which can be expressed in terms of complete elliptic integrals but it can also be approximated by simpler expressions ${ }^{25}$. Also : $t(y)=v(y)-\frac{2}{3} y \frac{d v(y)}{d y}$. Fig. 6 shows the results of these models for two couples of input data: $\varphi=4.25 \mathrm{eV}, \beta=1$ and $\varphi=4.02 \mathrm{eV}, \beta=1.5$, which correspond to the flat surface and to the corrugated surface near the ridge. As is well known, the attractive charge-image interaction reduces the tunneling barrier and enhances the emitted current density in the MG model which includes it, as compared to the FN one which neglects it. In the present case, saturation is reached for the MG model, especially 
Publishiwgen $\beta=1.5$. The results of our numerical model are larger than the FN ones and smaller than the MG ones. A similar result has already been observed for instance in the one dimensional DFT model of ref. 37 (see fig. 4 there). Interestingly, the FN current density for $\varphi=4.02 \mathrm{eV}, \beta=1.5$ is very close to our averaged density in the corrugated case. This may be fortuitous, the FN density is expected at best to model the one at the ridge. The slope of the FN plots is directly related to the work function and local field enhancement and is given by $\frac{4}{3 e}\left(\frac{2 m}{\hbar^{2}}\right)^{\frac{1}{2}} \frac{\varphi^{\frac{3}{2}} v\left(y_{F}\right)}{\beta}$ in the FN and MG models. These analytical models reproduce reasonably well the slopes of our numerical results : in particular, due to a larger field enhancement near the ridge and slightly smaller work function, the decrease of the emitted is smaller in the corrugated case than in the flat one in both numerical and analytical models. Similarly, in both types of models, the slope of the current density is smaller near the trough than near the ridge where the field enhancement is smaller.

Although the global emitted current density from the FN analytical model and our numerical model look qualitatively similar, they differ significantly if more detailed observables like emitted current density energy distribution (CDED) are considered. In the FN type models, the ED is given by (eq. 7 in ref. 20$)$ :

$$
\frac{d J}{d \epsilon}=\frac{e m\left(\epsilon_{F}-\epsilon\right)}{2 \pi^{2} \hbar^{3}} \exp \left(-\frac{4}{3 e}\left(\frac{2 m}{\hbar^{2}}\right)^{\frac{1}{2}} \frac{\left(\varphi+\epsilon_{F}-\epsilon\right)^{\frac{3}{2}} v(y)}{\beta F}\right)
$$

It is shown on fig. 7. The increase of the CDED (as energy increases) far below the Fermi level is controlled by the tunnel transmission, and its decrease close to Fermi level by the decrease of the density of states of the 2 dimensional free electron gaz describing the metal electrons. The CDED is shown for 2 field strengths, $15 \mathrm{~V} / \mathrm{nm}$ and $4 \mathrm{~V} / \mathrm{nm}$. For $4 \mathrm{~V} / \mathrm{nm}$, the distribution corresponds of course to lower amplitudes due to weaker tunneling, but is also more confined in energy in the vicinity of the Fermi level.

In our numerical model, the continuum of electron metal states is discretized and the energy distribution becomes a set of points $\left(\epsilon_{m}, \bar{J}_{m}\right)$, each associated to an orbital $\Psi_{m}(\mathbf{r}, \mathbf{F})$ (eq. 1). This set of points is shown on fig. 7 for the external field $15 \mathrm{~V} / \mathrm{nm}$. The current densities have been premultiplied by the degeneracy of the states. The total current density is thus the sum of the 192 individual densities shown on the figure. This distribution has two striking features. First, its shape resembles more the FN CDED for $4 \mathrm{~V} / \mathrm{nm}$ than for $15 \mathrm{~V} / \mathrm{nm}$. This may be related to the fact that the field experienced by the metal electrons is much smaller than $15 \mathrm{~V} / \mathrm{nm}$ in the vicinity of the metal, as shown on fig. $3:$ the external 
Publishifing is screened by a space charge layer of the order of $3 \AA$ thick. It is this screened field that the electrons experience when tunneling away from the metal.

The second striking feature of the CDED is the great variability of the state current density on energy : in a narrow energy band, the state current density can fluctuate from almost 0 to large values. This means that characteristics of the states $\left|\Psi_{m}(\mathbf{r}, \mathbf{F})\right\rangle$ different from their energy must be important factors influencing the current density level. To investigate this further, we consider for the flat surface case on fig. 8 and fig. 9 the projections of the states $\mid \Psi_{m}(\mathbf{r}, \mathbf{F}=0)>$ on selected orbitals. We consider only orbitals centered on the atoms facing vacuum to probe the electron density in the vieinity of the metal-vacuum interface, which corresponds to the area expected to have the largest influence on emission. We show on fig. 8 the projections on the orbitals pointing along $\mathrm{z}$ toward vacuum $\left(p_{z}\right.$ and $d_{z^{2}}$ spherical harmonics) on fig. 9 the projections on those pointing toward the surface $\left(p_{x}\right.$ and $d_{x y}$ spherical harmonics). The orbitals pointing toward vacuum are expected to transfer electrons from the metal to vacuum and enhance electron emission, whereas the ones pointing toward the surface are expected to confine the electrons in the metal. The results shown on fig. 8 and 9 confirm this hypothesis : there is a correlation between state current density and the weight of the contribution of the $p_{z}$ and $d_{z^{2}}$ orbitals to the emitting state, and on the contrary an anti-correlation for the $p_{x}$ and $d_{x y}$ cases, as indicated by the signs of the slopes of the linear fits shown on these figures. As the weights of the z-pointing orbitals differ greatly from one state to another one even in a narrow energy interval, the emitted current can vary greatly between states even close in energy. Such fine detailed properties of the emitting states cannot be accounted for in the simple FN type models.

\section{CONCLUSION}

We have studied the effect of the presence nanoscale steps on the electronic emission of a tungsten surface using a three dimensional quantum mechanical model using periodic DFT (VASP) coupled to a perturbation theory wavepacket propagation method. The orders of magnitude of the averaged current density emitted from the corrugated surface are similar to the ones obtained from the flat one. However, strong enhancements are obtained on local current densities near the edges of the steps.

Both the present numerical DFT type models and the FN type analytical ones thus 
Publishijpgrgide results in qualitative agreement, but they have different strengths and weaknesses. The DFT type models have the advantage to provide results relying only on the definition of the emitting structure at the atomic level, without any additional adjustable parameter. From this point of view, they provide ab-initio emitted current densities. This class of methods has a large flexibility of applications : it has been used here to study the effect of corrugation on emission, but it could be used as well to study other problems such as the effect of contaminants on surfaces ${ }^{18}$ or anomalous emission properties from 2D materials ${ }^{30}$. On the other hand, in many engineering applications, the-emitting structure is not fully controlled at the atomic level and it is difficult to obtain valuable information from DFT models in this context. The FN type analytical models are then still very valuable in that they allow to grasp an essential part of the physics of the problem with a few parameters.

\section{ACKNOWLEDGEMENTS}

We thank Dr. M. Márquez-Mijares for an in-depth reading of the manuscript and useful comments.

* Electronic address: bruno.lepetit@irsamc.ups-tlse.fr

1 A. Simonin, J. Achard, K. Achkasov, S. Bechu, C. Baudouin, O. Baulaigue, C. Blondel, J. Boeuf, D. Bresteau, G. Cartry, et al., Nucl. Fusion 55, 123020 (2015).

2 H. P. L. de Esch, A. Simonin, C. Grand, B. Lepetit, D. Lemoine, and M. Márquez-Mijares, AIP Proc. 1869, 060003 (2017).

3 R. H. Fowler and L. Nordheim, Proceedings of the Royal Society of London A: Mathematical, Physical and Engineering Sciences 119, 173 (1928).

4 E. L. Murphy and R. H. Good, Physical Review 102, 1464 (1956).

5 A. Simonin, H. d. Esch, L. Doceul, L. Christin, F. Faisse, and F. Villecroze, Fusion Engineering and Design 88, 1 (2013).

6 R. S. Hemsworth, A. Tanga, and V. Antoni, Review of Scientific Instruments 79, 02C109 (2008).

7 R. Hemsworth, H. Decamps, J. Graceffa, B. Schunke, M. Tanaka, M. Dremel, A. Tanga, H. P. L. de Esch, F. Geli, J. Milnes, et al., Nuclear Fusion 49, 045006 (2009). 
Publishing̊gR. V. Latham, High Voltage Vacuum Insulation: Basic Concepts and Technological Practice (Elsevier, New-York, 1995).

9 D. Alpert, D. Lee, E. M. Lyman, and H. E. Tomaschke, Journal of Applied Physics 38, 880 (1967).

10 G. Beukema, Physica 61, 259 (1972).

11 R. N. Bloomer and B. M. Cox, Vacuum 18, 379 (1968).

12 B. Bonin, Vacuum 46, 907 (1995).

13 A. Zeitoun-Fakiris and B. Juttner, Journal of Physics D: Applied Physics 21, 960 (1988).

14 Y. Yamamoto and T. Miyokawa, Journal of Vacuum Science and Technology B (Microelectronics and Nanometer Structures) 16, 2871 (1998).

15 K. Almousa Almaksour, Ph.D. thesis (2014), URL http://www.theses.fr/2014PA112017/ document.

16 K. Almaksour, M. J. Kirkpatrick, P. Dessante, E. Odic, A. Simonin, H. P. L. de Esch, B. Lepetit, D. Alamarguy, F. Bayle, and P. Teste, Rhys. Rev. ST Accel. Beams 17, 103502 (2014).

17 M. Márquez-Mijares, B. Lepetit, and D. Lemoine, Surface Science 645, 56 (2016).

18 M. Márquez-Mijares, B. Lepetit, D. Lemoine, K. Almaksour, M. J. Kirkpatrick, P. Dessante, E. Odic, D. Alamarguy, F. Bayle, P. Teste, et al., J. Vac. Sci. Technol. B 34, 061208 (2016).

19 B. Lepetit, D. Lemoine, and M. Márquez-Mijares, J. App. Phys. 120, 085105 (2016).

20 B. Lepetit, J. Appl. Phys. 122, 215105 (2017).

21 L. W. Nordheim, Proc. R. Soc. London, Ser. A 121, 626 (1928).

22 R. Burgess, H. Kroemer, and J. Houston, Phys. Rev. 90, 515 (1953).

23 J. He, P. H. Cutler, and N. M. Miskovsky, App. Phys. Lett. 59, 1644 (1991).

24 A. Modinos, Solid-State Electronics 45, 809 (2001).

25 R. G. Forbes, App. Phys. Lett. 89, 113122 (2006).

26 R. G. Forbes, J. H. B. Deane, A. Fischer, and M. S. Mousa, Jordan Journal of Physics 8, 125 (2015)

27 R. G. Forbes and J. H. B. Deane, Proc. R. Soc. London A 467, 2927 (2011).

28 Y. Li, Y. Sun, and J. Yeow, Nanotechnology 26, 242001 (2015).

29 Z. B. Li, Ultramicroscopy 159, 162 (2015).

30 Y. Ang, S.-J. Liang, and L. Ang, MRS Bulletin 42, 505 (2017).

31 Y. Ang, M. Zubair, K. Ooi, and L. Ang, ArXiv : 1711.05898v1 (2018). 
Publishîîigs. Han, M. H. Lee, and J. Ihm, Phys. Rev. B 65, 085405 (2002).

33 Y.-W. Son, S. Oh, J. Ihm, and S. Han, Nanotechnology 16, 125 (2005).

34 C. Choong-Ki Lee, B. Lee, and S. Ihm, J. Han, Nanotechnology 18, 475706 (2007).

35 J. Peng, Z. Li, C. He, G. Chen, W. Wang, S. Deng, N. Xu, X. Zheng, G. Chen, C. J. Edgcombe, et al., Journal of Applied Physics 104, 014310 (2008).

36 M. Khazaei, A. A. Farajian, and Y. Kawazoe, Phys. Rev. Lett. 95, 177602 (2005).

37 Y. Gohda, Y. Nakamura, K. Watanabe, and S. Watanabe, Phys. Rev. Lett. 85, 1750 (2000).

38 Y. Gohda and S. Watanabe, Phys. Rev. Lett. 87, 177601(2001).

39 Y. Gohda and S. Watanabe, Surface Science 516, 265 (2002).

40 Y. Gohda, Y. Nakamura, K. Watanabe, and S. Watanabe, Materials Science and Engineering A 327, 1 (2002).

41 Y. Gohda and S. Watanabe, J. Phys.: Condens. Matter 16, 4685 (2004).

42 A. Mayer, N. M. Miskovsky, P. H. Cutler, and P. Lambin, Phys. Rev. B 68, 235401 (2003).

43 S. F. Huang, T. Leung, B. Li, and C. Chan, Phys. Rev. B 72, 035449 (2005).

44 P. Yaghoobi, K. Walus, and A. Nojeh, Phys. Rev. B 80, 115422 (2009).

45 Z. Li, N. Xu, and H. J. Kreuzer, Phys. Rev. B 85, 115427 (2012).

46 R. V. Latham, High Voltage Vacuum Insulation: a new perspective (AuthorHouse, 2006).

47 M. Zubair, Y. S. Ang, and L. K. Ang, IEEE Trans. Elec. Devices 65, 2089 (2018).

48 B. M. Cox and W. T. Williams, Journal of Physics D: Applied Physics 10, L5 (1977).

49 C. Suzuki, T. Nakanishi, S. Okumi, T. Gotou, K. Togawa, F. Furuta, K. Wada, T. Nishitani, M. Yamamoto, J. Watanabe, et al., Nuclear Instruments and Methods in Physics Research Section A: Accelerators, Spectrometers, Detectors and Associated Equipment 462, 337 (2001).

50 F. Furuta, T. Nakanishi, S. Okumi, T. Gotou, M. Yamamoto, M. Miyamoto, M. Kuwahara, N. Yamamoto, K. Naniwa, K. Yasui, et al., Nuclear Instruments and Methods in Physics Research Section A: Accelerators, Spectrometers, Detectors and Associated Equipment 538, 33 $(2005)$.

51 A. Dangwal Pandey, G. Müller, D. Reschke, and X. Singer, Phys. Rev. ST Accel. Beams 12, 023501 (2009).

52 R. G. Forbes, C. Edgcombe, and U. Valdrè, Ultramicroscopy 95, 57 (2003).

53 C. Kittel, Introduction to solid state physics (Wiley, 1996), 7th ed.

54 J. Bardeen, Phys. Rev. Lett. 6, 57 (1961). 
Publishithy. Tersoff and D. R. Hamann, Phys. Rev. B 31, 805 (1985).

56 D. S. Sholl and J. A. Steckel, Density fucntional theory. A practical introduction (Wiley, 2009).

57 G. Kresse and J. Hafner, Physical Review B 47, 558 (1993).

58 G. Kresse and J. Hafner, Physical Review B 49, 14251 (1994).

59 G. Kresse and J. Furthmüller, Computational Materials Science 6, 15 (1996).

60 G. Kresse and J. Furthmüller, Physical Review B 54, 11169 (1996).

61 P. E. Blöchl, Physical Review B 50, 17953 (1994).

62 G. Kresse and D. Joubert, Physical Review B 59, 1758 (1999).

63 J. P. Perdew, K. Burke, and M. Ernzerhof, Physical Review Letters 77, 3865 (1996).

64 J. P. Perdew, K. Burke, and M. Ernzerhof, Physical Review Letters 78, 1396 (1997).

65 Y. Zhang and W. Yang, Physical Review Letters 80, 890 (1998).

66 M. Methfessel and A. Paxton, Physical Review B 40, 3616 (1989).

67 R. M. Feenstra and M. Widom, waveTrans : real-space wavefunctions from VASP Wavecar file, URL http://www . andrew. cmu . edu/user/feenstra/wavetrans/.

68 P. J. Jennings, R. O. Jones, and M. Weinert, Phys. rev. B 37, 6113 (1988).

69 P. A. Serena, J. M. Soler, and N. Garcia, Phys. Rev. B 34, 6767 (1986).

70 R. Ramprasad, L. R. C. Fonseca, and P. von Allmen, Phys. Rev. B 62, 5216 (2000).

71 O. Gunnarsson, M. Jonson, and B. I. Lundqvist, Phys. Rev. B 20, 3136 (1979).

72 O. Gunnarsson and R. O. Jones, Phys. Scripta 21, 394 (1980).

73 A. G. Eguiluz, M. Heinrichsmeier, A. Fleszar, and W. Hanke, Phys. Rev. Lett. 68, 1359 (1992).

74 P. García-González and R. W. Godby, Comput. Phys. Com. 137, 108 (2001).

75 M. D. Feit, J. A. Fleck, and A. Steiger, J. Comput. Phys. 47, 412 (1982). 


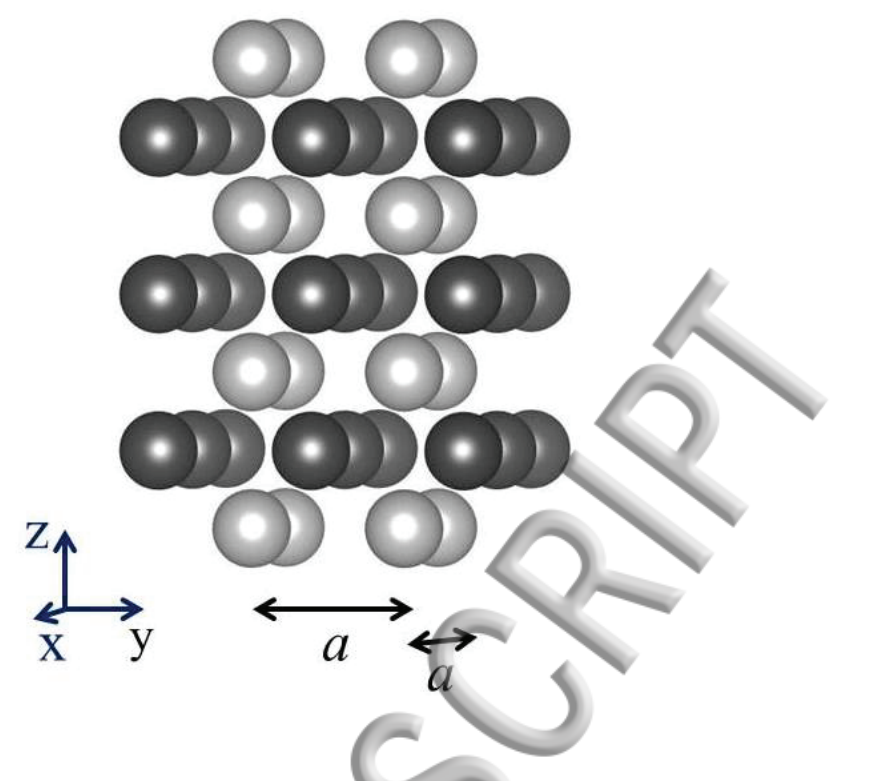

FIG. 1: The flat tungsten structure considered in the present study. It is a slab consisting in the superposition of 3 cubic cells (lattice parameter $a$ ) along one direction, but infinitely (only twice on the figure) repeated by periodicity along the two others. The slab thus consists of 7 atomic planes, separated by a distance $\frac{a}{2}$. Light and dark gray colors are used to help distinguishing tungsten atoms belonging to successive layers. The external field $\mathbf{F}$ is applied on this structure parallel to the $z$ axis chosen perpendicular to the slab. 
This manuscript was accepted by J. Appl. Phys. Click here to see the version of record.
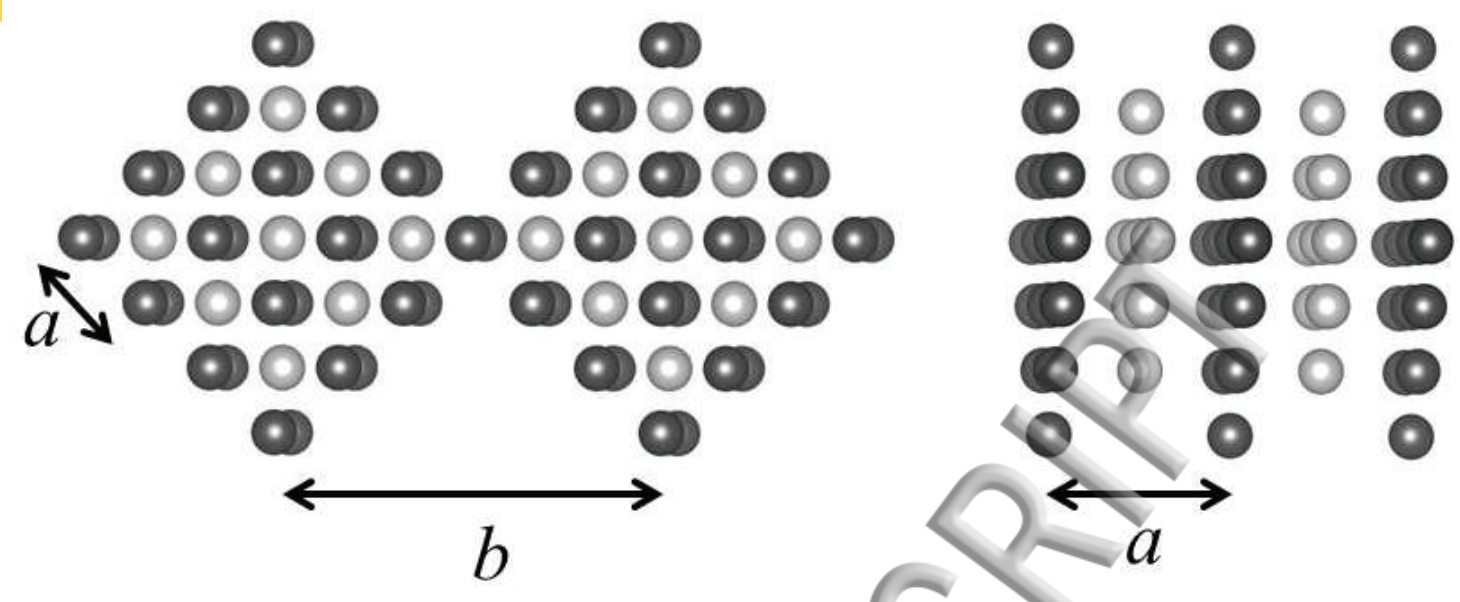

-

FIG. 2: The corrugated tungsten structure considered in the present study. It is bounded by $(101)$ surfaces consisting of $3(001) \times 3(100)$ terraces $\times$ steps. The slab is symetrized with respect to a mid-plane to facilitate convergence of the DFT calculation. A minimum thickness has been chosen for the structure to ease computational convergence. The slab is periodic, with periods $b=3 \sqrt{2} a$ and $a$. Light and dark gray colors are used to help distinguishing tungsten atoms belonging to successive layers. The external field $\mathbf{F}$ is applied on this structure parallel to the $z$ chosen perpendicular to the slab. 


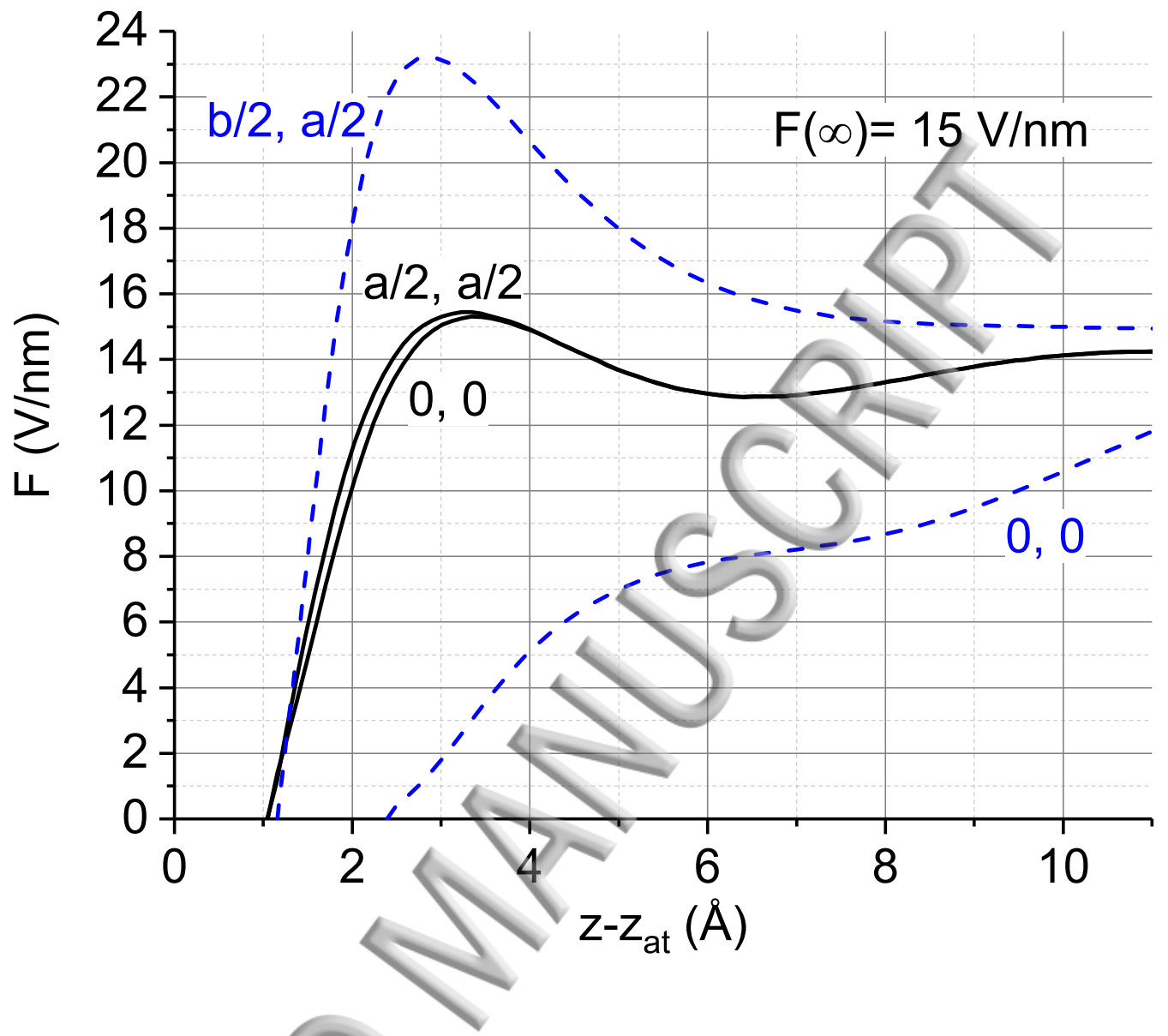

FIG. 3: $z$ component of the induced electric field as a function of the height $z-z_{a t}$ in the vicinity of the flat (full lines) and corrugated (dashed lines) surfaces. The far field amplitude is $15 \mathrm{~V} / \mathrm{nm}$. The chosen $x, y$ locations are at the corner $(=0,0)$ and the center $(=a / 2, a / 2$ and $=b / 2, a / 2$ for the flat and the corrugated structures, respectively) of the unit cell. The reference height, $z_{a t}$, depends on the chosen line of observation. In the flat case, it is the height of the topmost atomic layer. In the corrugated case, it is the height of the ridge for $x, y=b / 2, a / 2$, and it is the height of the troughs for $x, y=0,0$. The height of the ridge is defined as the one of the topmost atom for $x, y=b / 2,0$ and the height of the trough as the one of the topmost atom for $x, y=0,0$. 


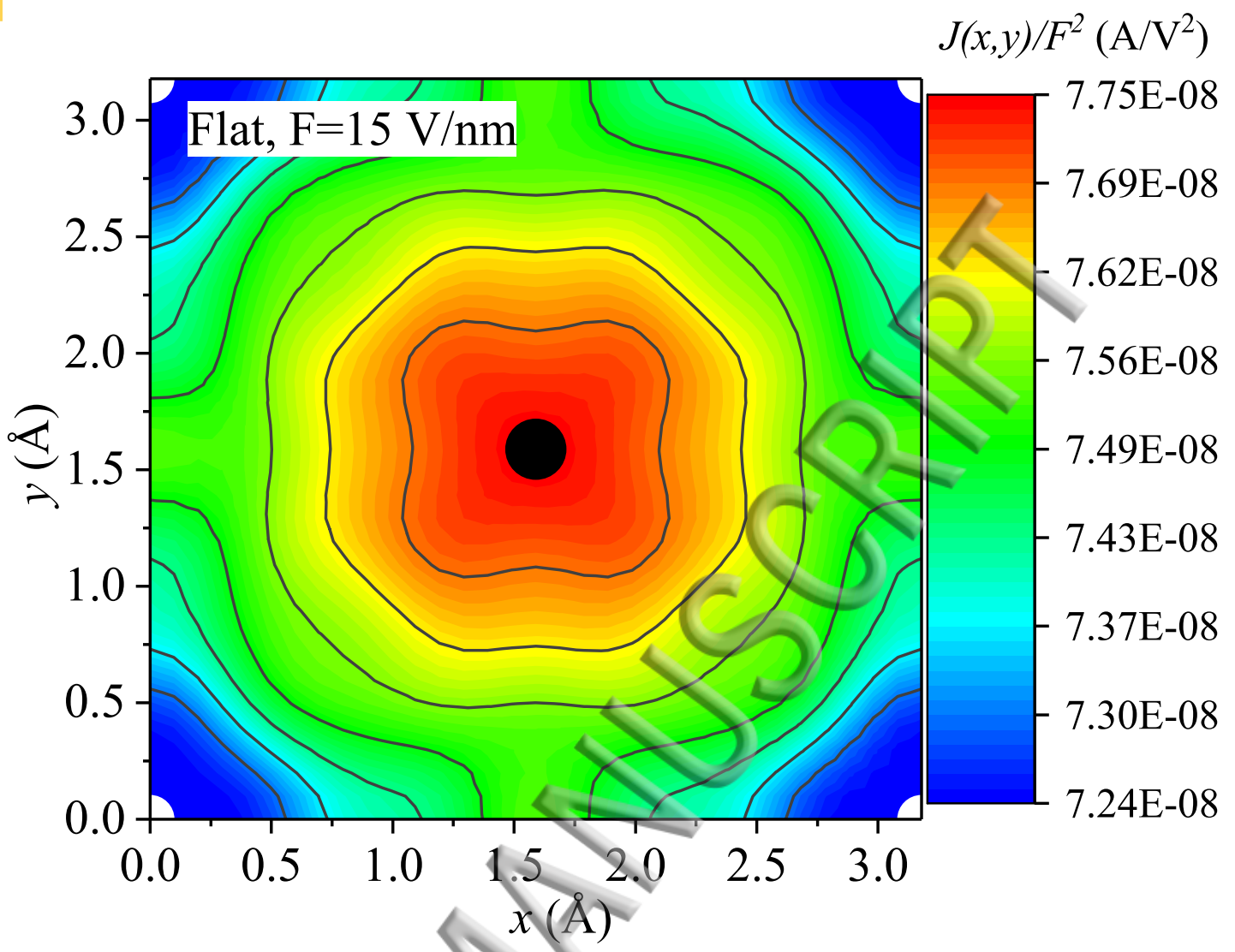

FIG. 4: Scaled local current density $J(x, y) / F^{2}$ (unit : $\mathrm{A} / \mathrm{V}^{2}$ ) for the flat structure of fig. 1 submitted to an external field $F=15 \mathrm{~V} / \mathrm{nm}$. The figure is restricted to the square $a \times a$ unit cell, $a=3.179 \AA$. The central black disk represents the location of the topmost layer atom, the 4 white sectors at the corners the 4 atoms belonging to the first sub-surface layer. The distance between these 2 layers is $\frac{a}{2}$. The scale at the right of the figure gives the minimum and maximum values of $J(x, y) / F^{2}$ as well as the 7 contour line values. 


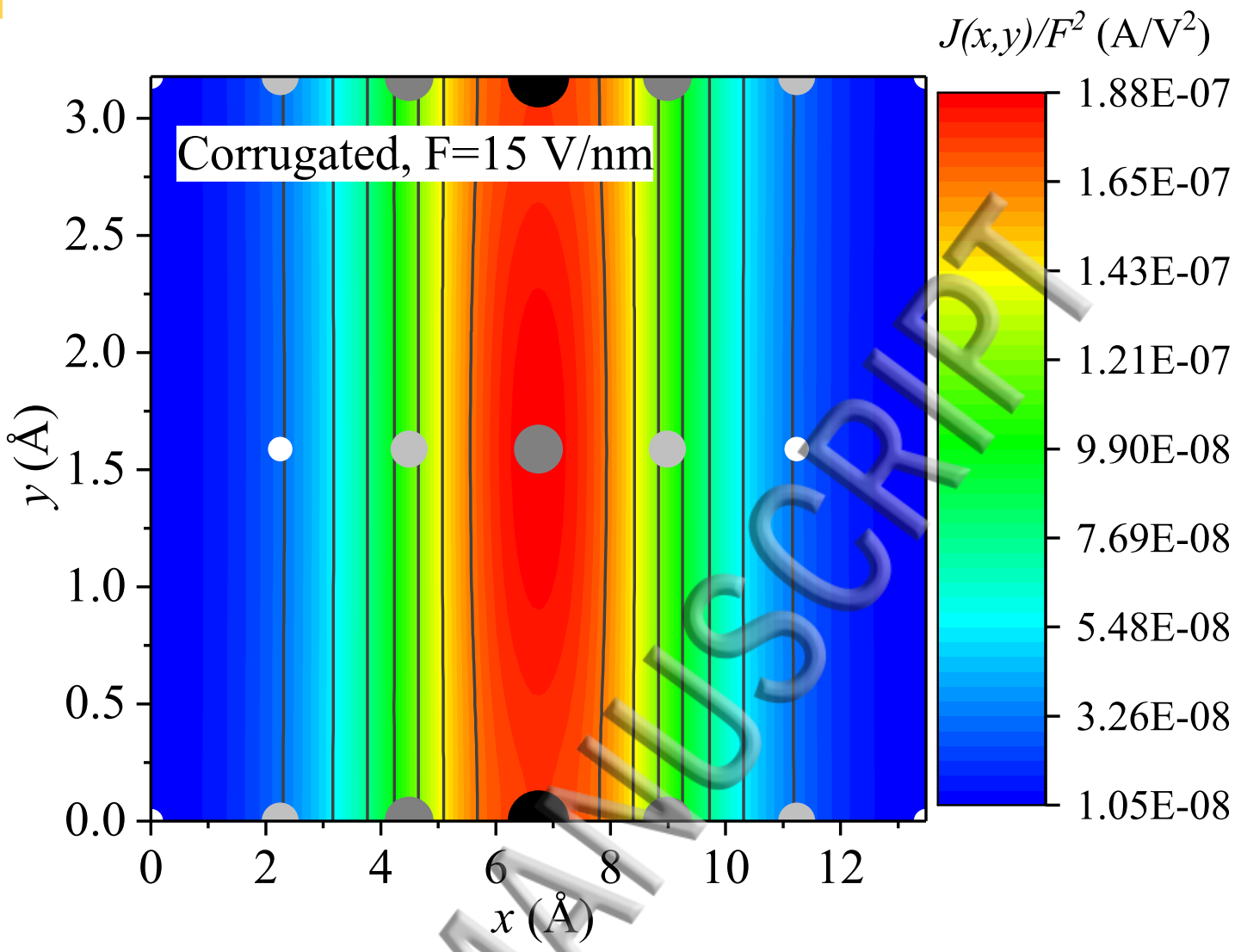

FIG. 5: Same as fig. 4 but for the corrugated structure of fig. 2. The figure is restricted to the rectangular $b \times a$ unit cell $b=3 \sqrt{2} a, a=3.179 \AA$. The disks represent the locations of the atoms facing vacuum, they belong to different atomic planes with different heights (the height between adjacent planes is $\left(\frac{a}{\sqrt{2}}\right)$. Atoms belonging to increasingly high planes are represented by increasingly dark and large disks. 


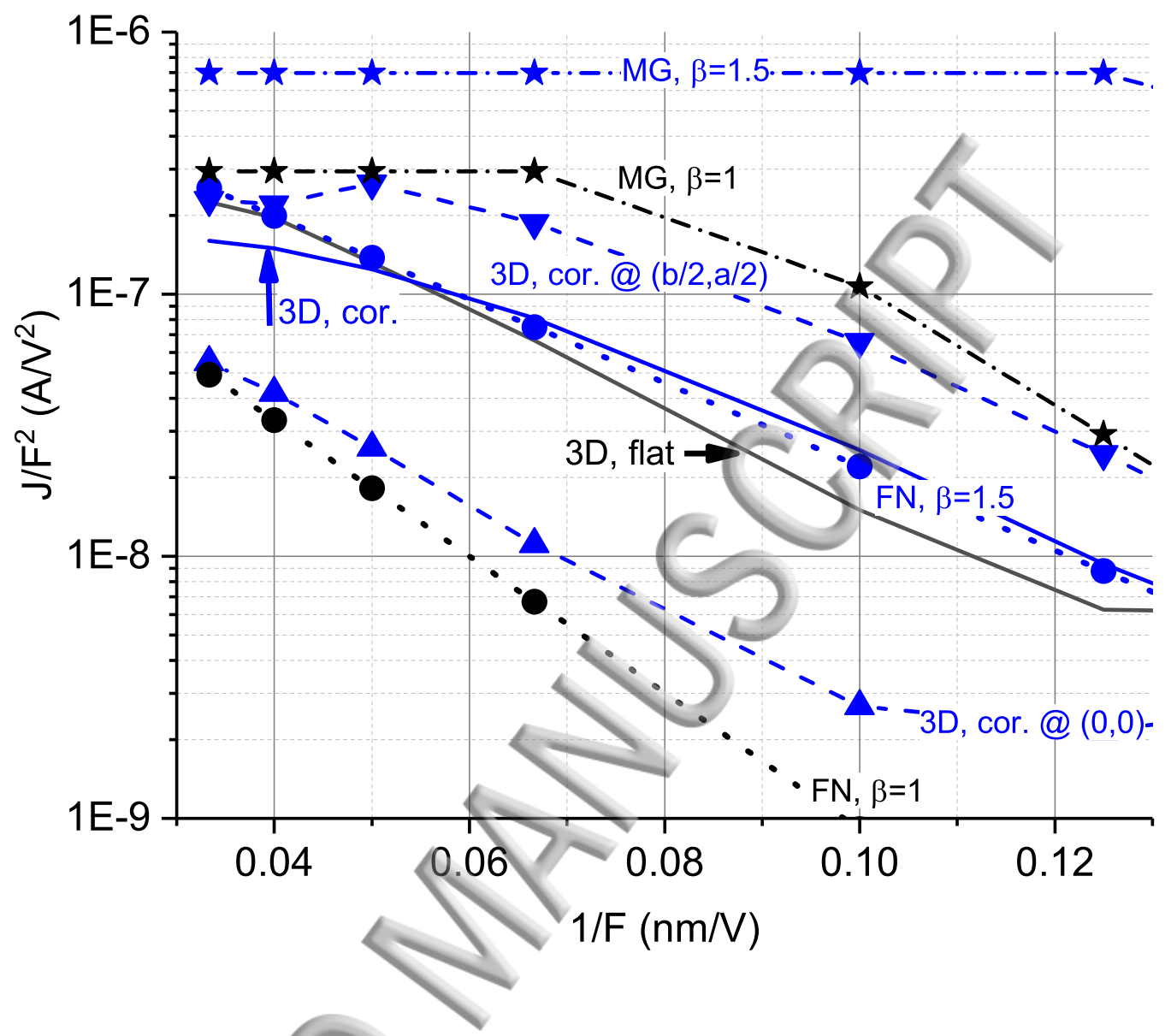

FIG. 6: Fowler-Nordheim plots of the field emitted current density from the flat (black curves) and corrugated (blue) structures. Full lines without markers : averaged emitted current density $\bar{J}$ resulting from the present numerical model for the flat and corrugated surfaces. Dashed lines with up and down pointing triangles : local current densities for the corrugated case at $(x, y)=(0,0)$ and $(x, y)=(b / 2, a / 2)$ respectively. Local densities are not shown for the flat structure because they do-not differ significantly from the averaged one in this case (see fig. 4). Dotted lines with disks : results of the Fowler-Nordheim model for the cases : work function $\varphi=4.25 \mathrm{eV}$, enhancement factor $\beta=1$ and $\varphi=4.02 \mathrm{eV}, \beta=1.5$; they are chosen to represent the flat and corrugated (respectively) cases. Dash-dotted lines with stars : results of the Murphy-Good model for $\varphi=4.25 \mathrm{eV}, \beta=1$ and $\varphi=4.02 \mathrm{eV}, \beta=1.5$. 


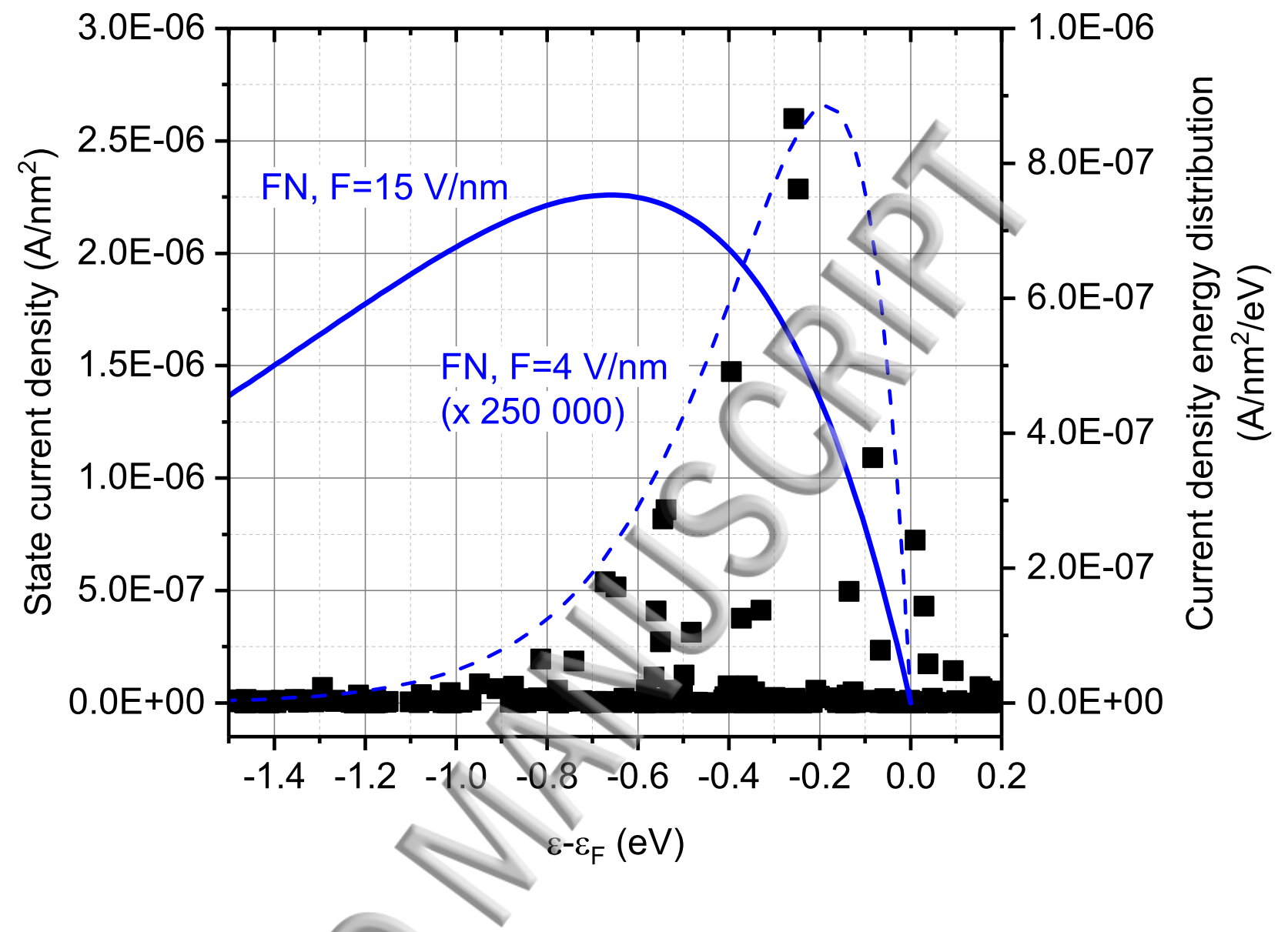

FIG. 7: Squares (left scale) : averaged state current density from our numerical model, as a function of the state energy, for the flat surface. These current densities include the degeneracy of each state. The external field is $15 \mathrm{~V} / \mathrm{nm}$. Full and dashed lines (right scale) : current density energy distribution (CDED) for the FN model. Full line : the external field is $15 \mathrm{~V} / \mathrm{nm}$, dashed line : the external field is $4 \mathrm{~V} / \mathrm{nm}$ and the energy distribution has been magnified by a factor 250000 . 


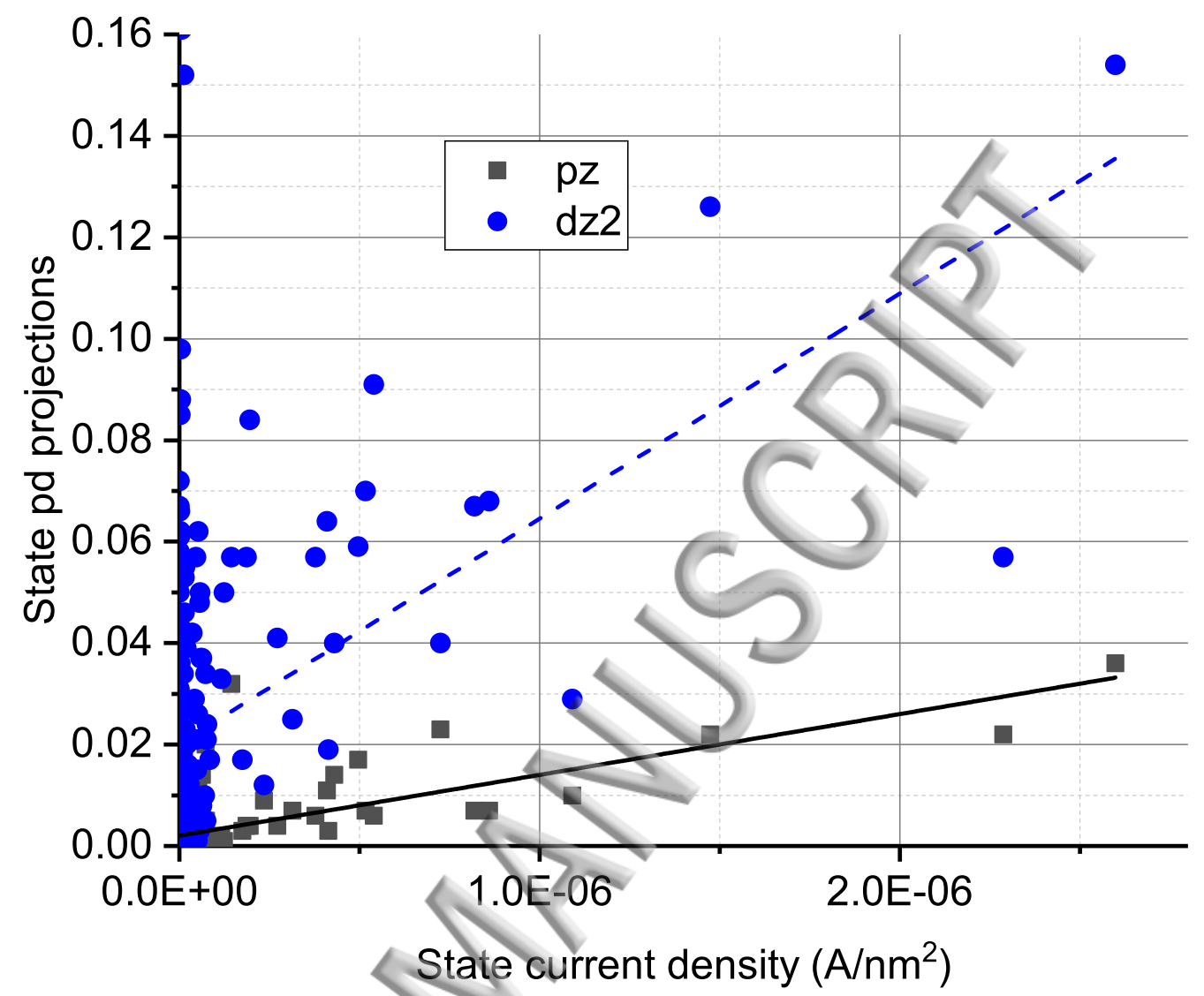

FIG. 8: Squares (left scale) : projections of the states $\mid \Psi_{m}(\mathbf{r}, \mathbf{F}=0)>$ on spherical harmonics centered on topmost atoms of the flat structure, as a function of the averaged state current density (degeneracy included). The projections are performed for the $p_{z}$ and $d_{z^{2}}$ spherical harmonics. The linear least square fits performed for each set of points are shown as full $\left(p_{z}\right)$ and dashed $\left(d_{z^{2}}\right)$ lines. 


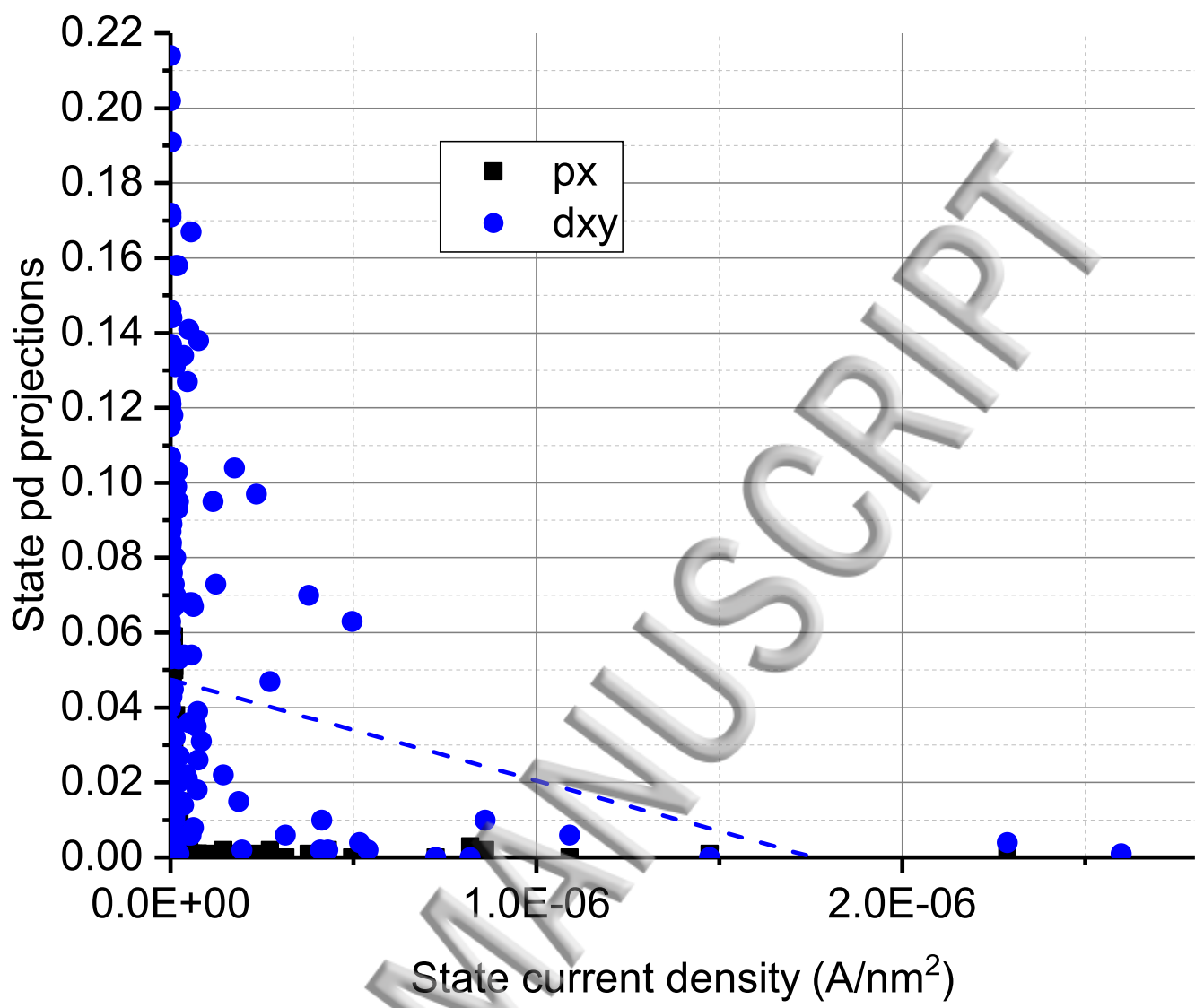

FIG. 9: Squares (left scale): same as fig. 8 , but the projection is now performed on the $p_{x}$ and $d_{x y}$ spherical harmonics. The linear least square fit is performed for the $d_{x y}$ set of points only, as the $p_{x}$ projections are comparatively negligible. 


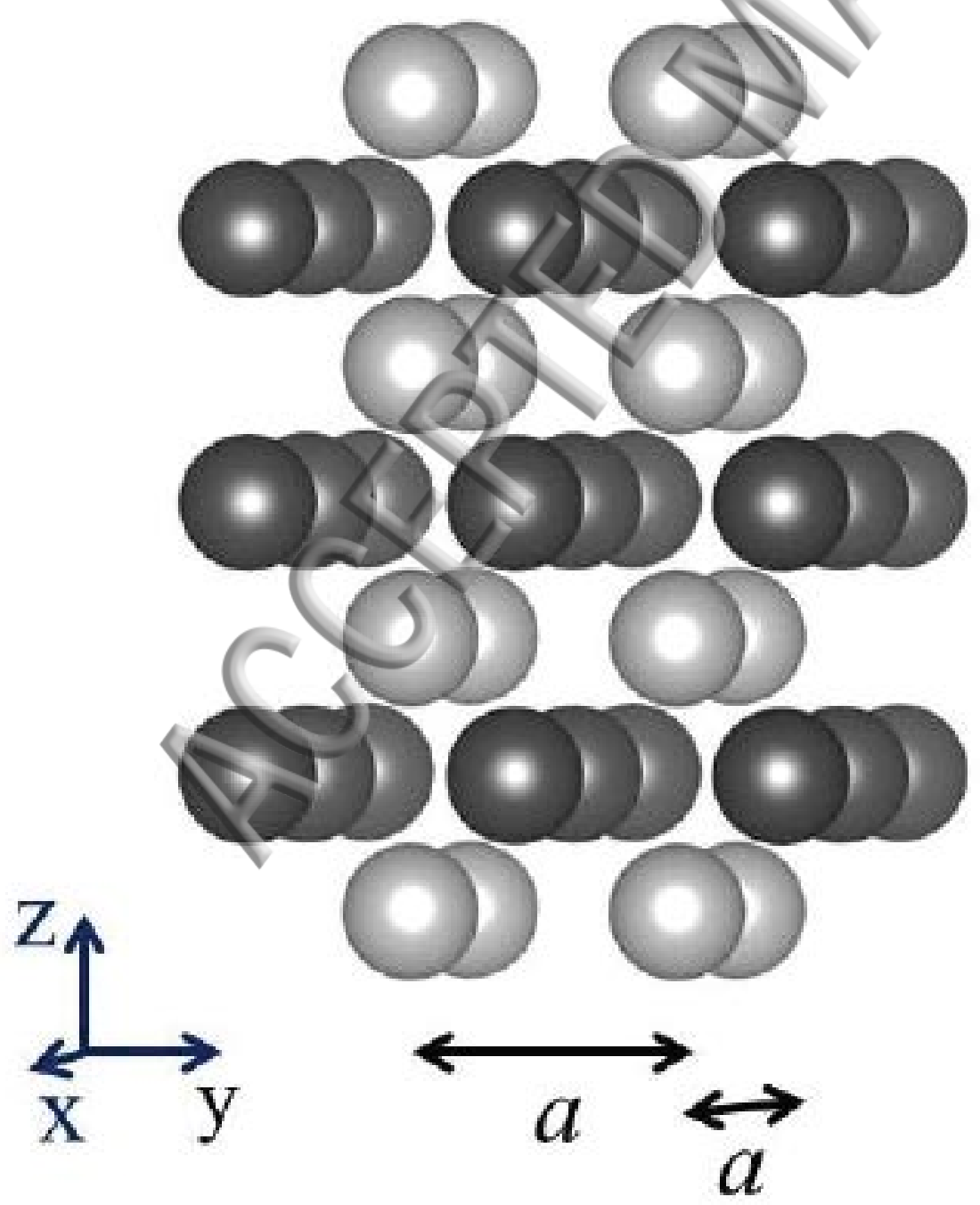




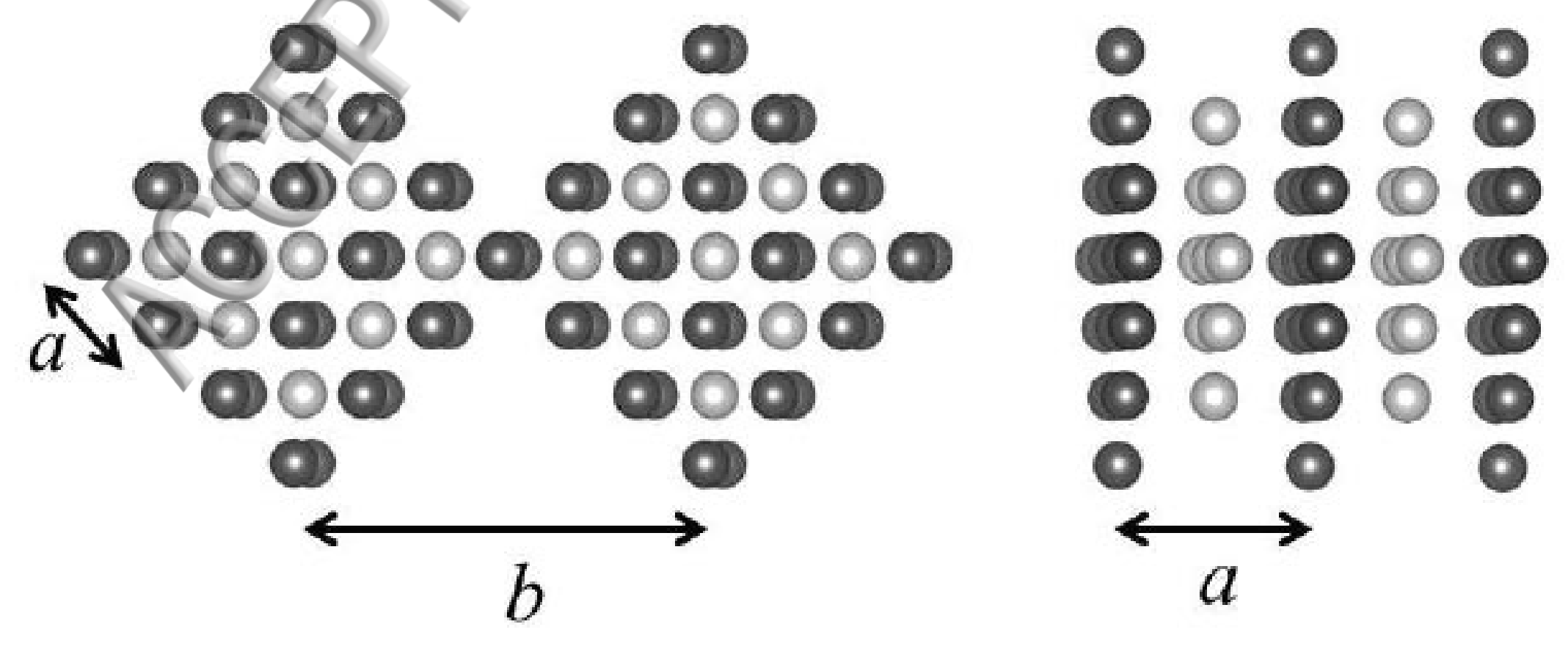




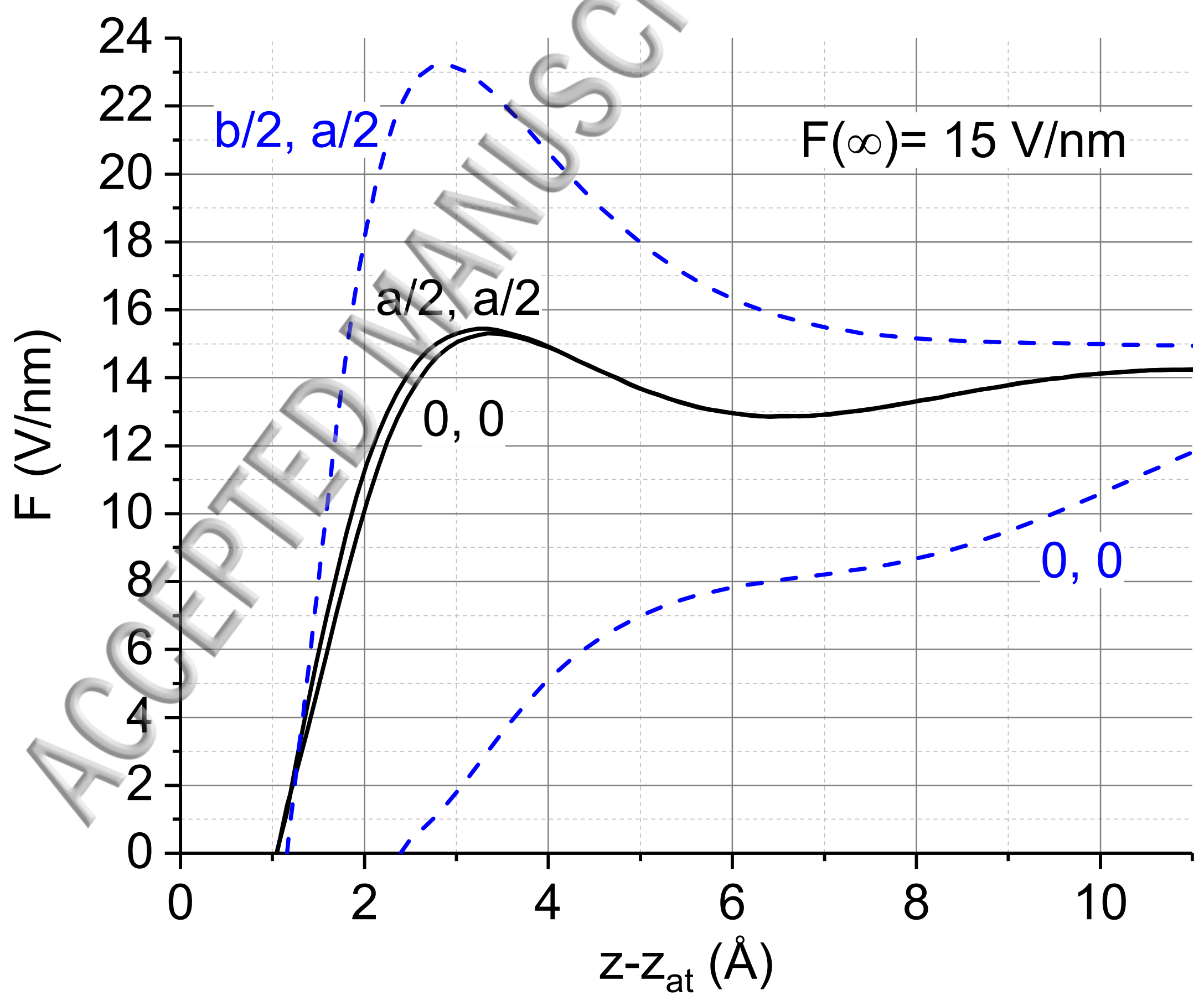




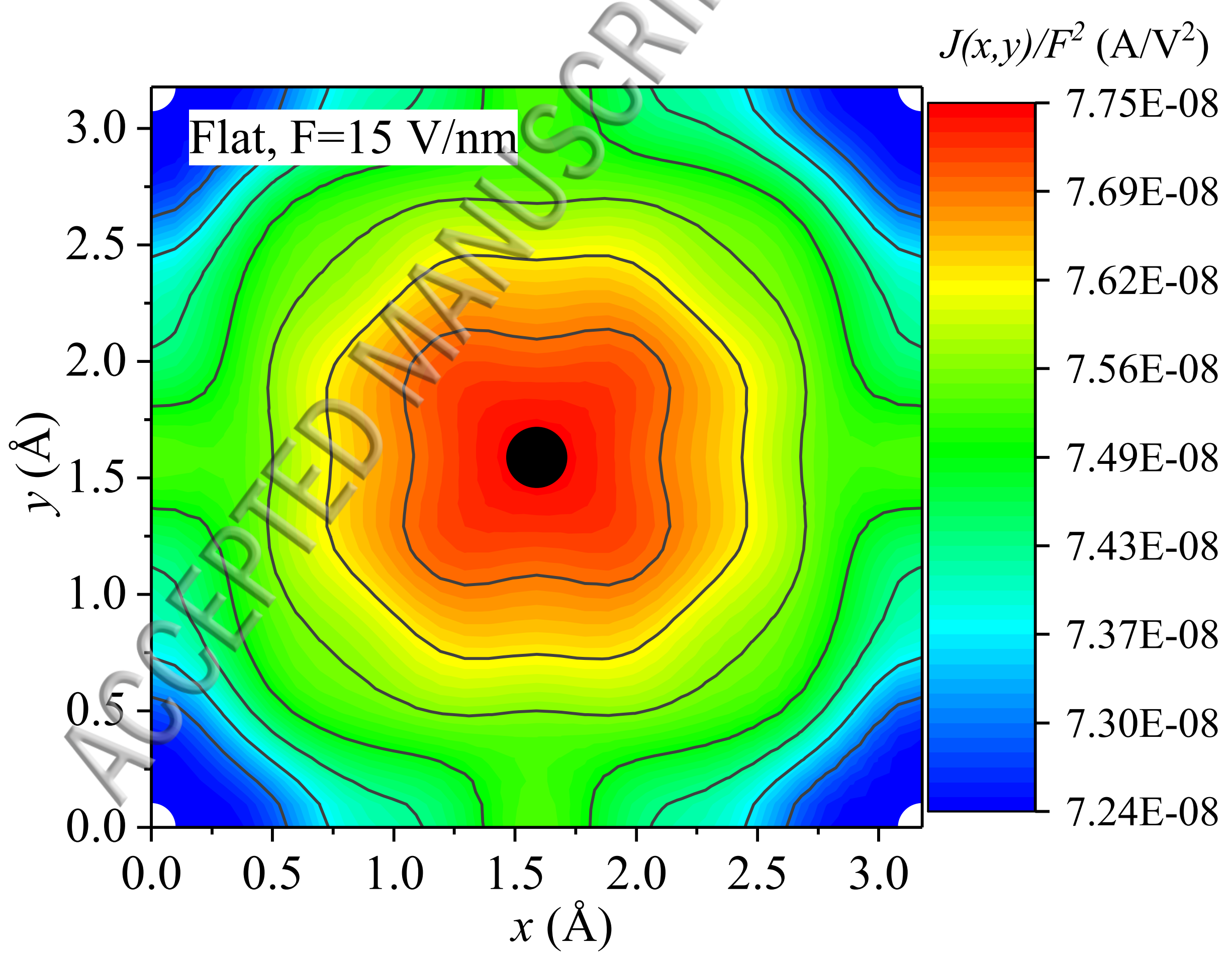


$J(x, y) / F^{2}\left(\mathrm{~A} / \mathrm{V}^{2}\right)$

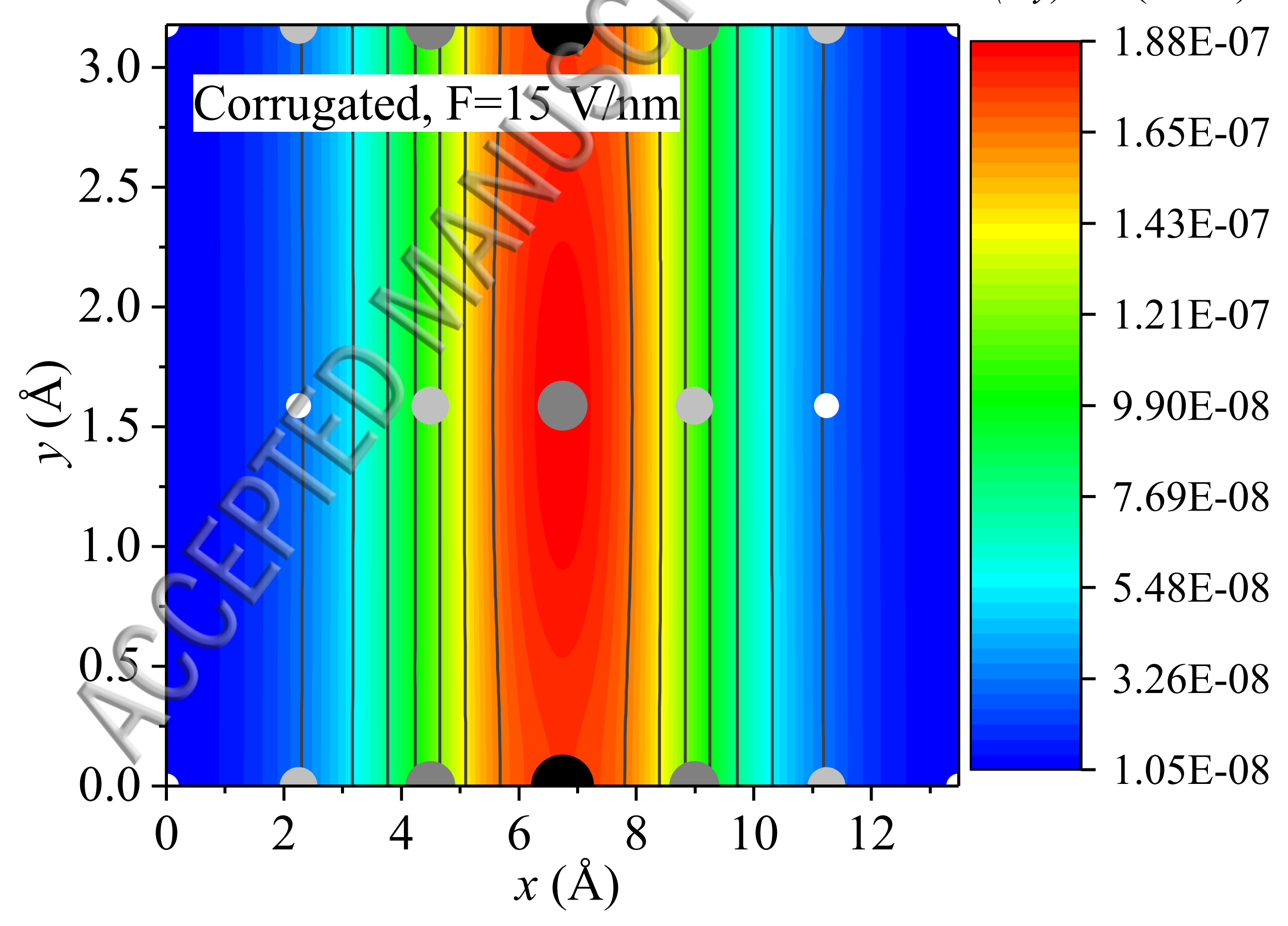




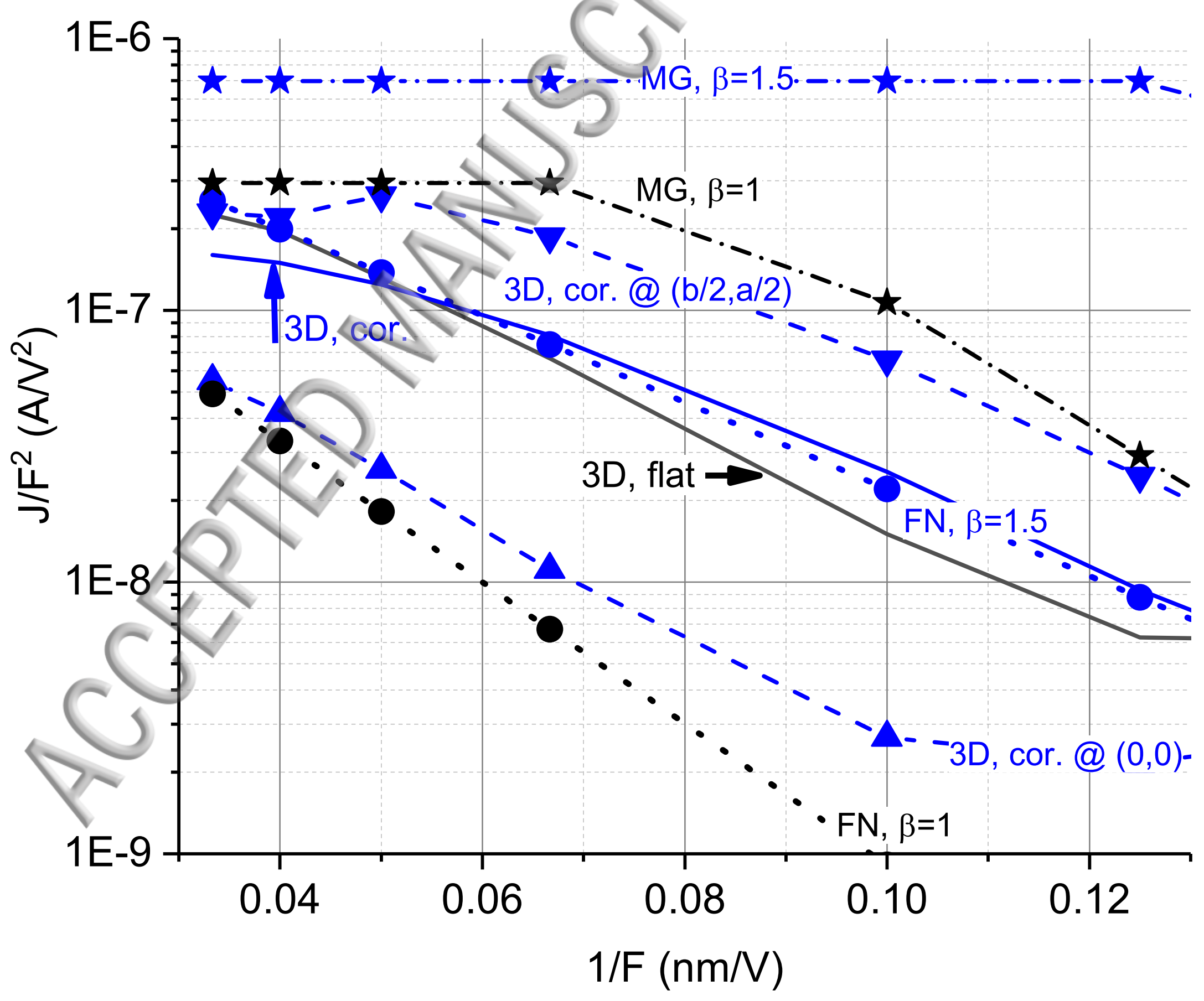




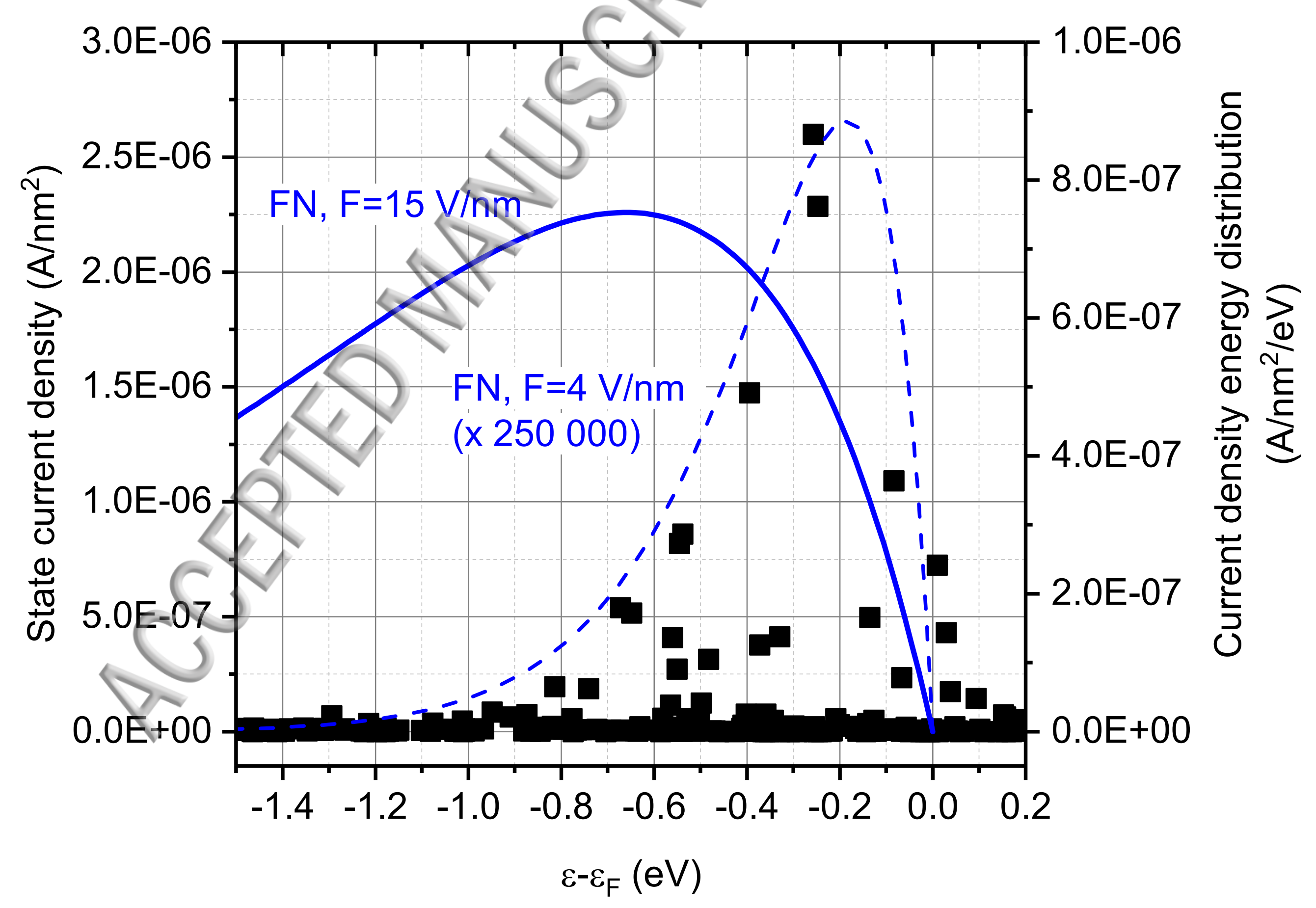




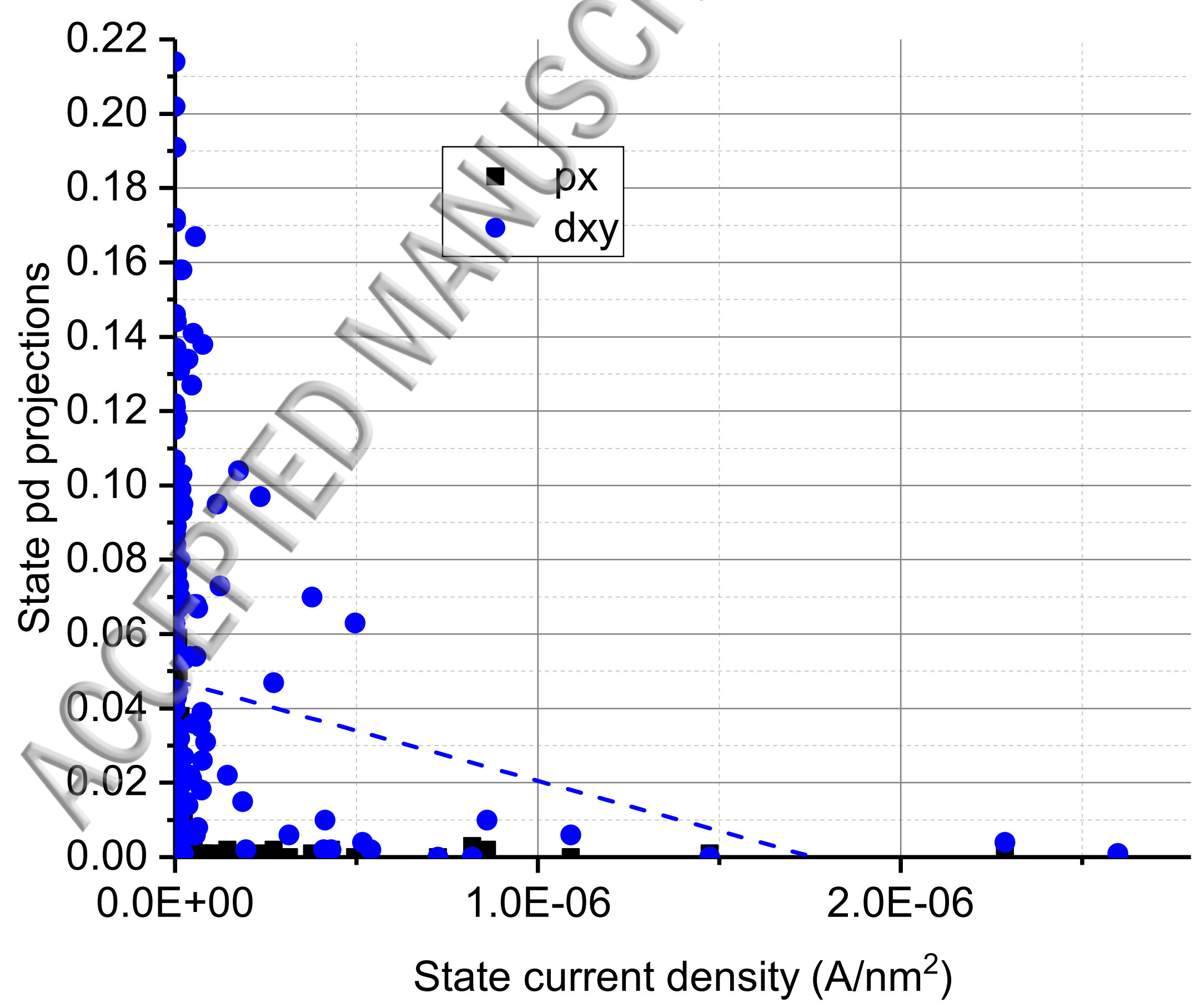




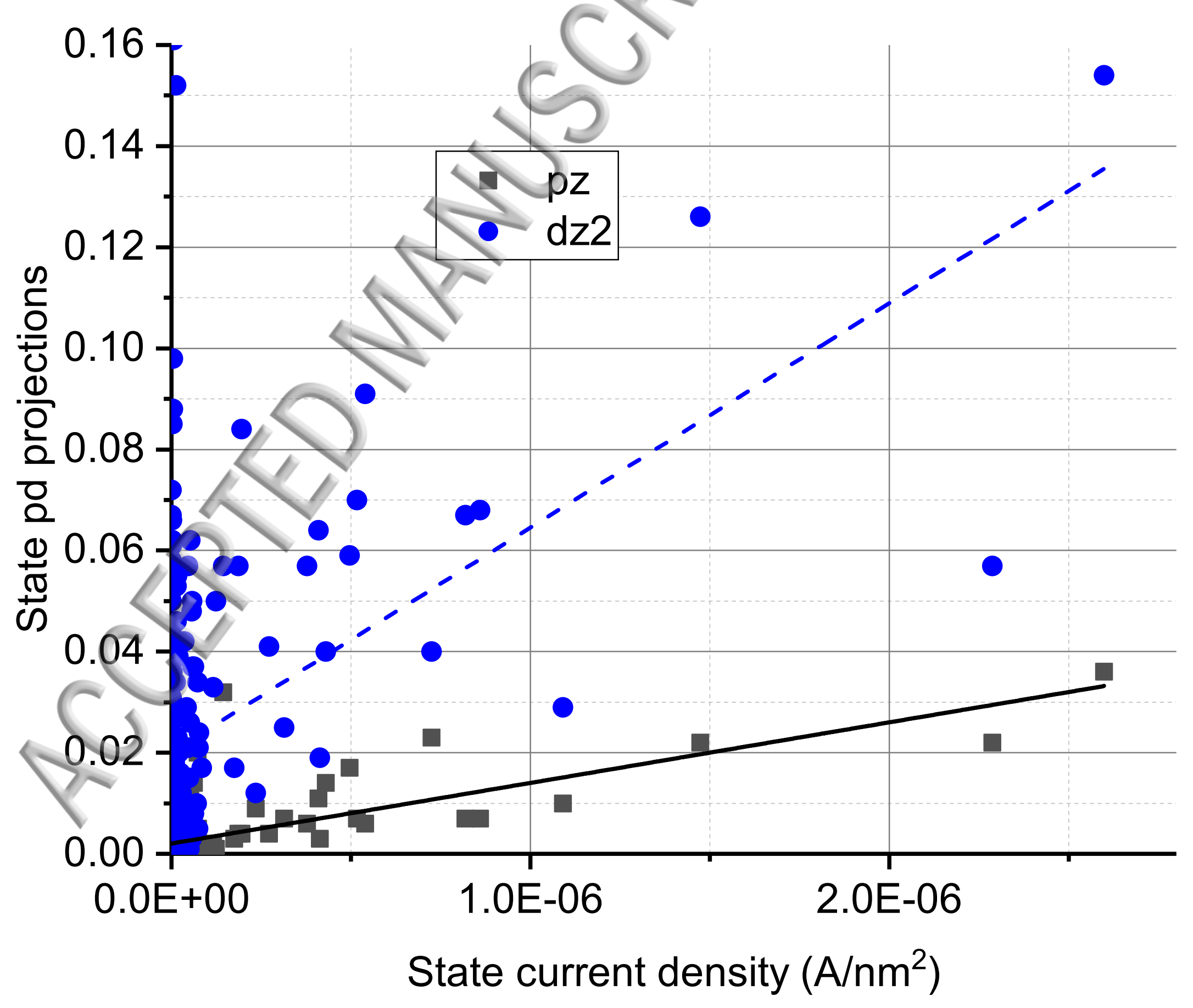

